Meta-analysis

\title{
Effects of preeclampsia and eclampsia on maternal metabolic and biochemical outcomes in later life: a systematic review and meta-analysis
}

\author{
Vanesa Alonso-Ventura ${ }^{\mathrm{a}}$, Yangzhou Li ${ }^{\mathrm{b}}$, Vinay Pasupuleti ${ }^{\mathrm{c}}$, Yuani M. Roman ${ }^{\mathrm{d}}$, \\ Adrian V. Hernandez ${ }^{\text {b,d,e }}$, Faustino R. Pérez-López ${ }^{\text {f,* }}$ \\ a Cardiology Department, Miguel Servet University Hospital, Zaragoza 50009, Spain \\ b University of Connecticut School of Pharmacy, Storrs, CT 06269, USA \\ c MedErgy HealthGroup Inc., Yardley, PA 19067, USA \\ d University of Connecticut/Hartford Hospital Evidence-based Practice Center, Hartford, CT 06102, USA \\ e School of Medicine, Universidad Peruana de Ciencias Aplicadas (UPC), Lima 9, Peru \\ ${ }^{\mathrm{f}}$ Department of Obstetrics and Gynecology, University of Zaragoza Faculty of Medicine, and Instituto de Investigaciones Sanitarias de Aragón, Zaragoza 50009, Spain
}

\section{A R T I C L E I N F O}

Article history:

Received 8 September 2019

Accepted 10 November 2019

\section{Keywords:}

Preeclampsia

Eclampsia

HELLP syndrome

Metabolic outcomes

Biochemical outcomes

Cardiovascular risk

Meta-analysis

\begin{abstract}
A B S T R A C T
Objective: To evaluate the association between preeclampsia (PE) and eclampsia (E) on subsequent metabolic and biochemical outcomes.

Methods: Systematic review and meta-analysis of observational studies. We searched five engines until November 2018 for studies evaluating the effects of PE/E on metabolic and biochemical outcomes after delivery. PE was defined as presence of hypertension and proteinuria at $>20$ weeks of pregnancy; controls did not have PE/E. Primary outcomes were blood pressure (BP), body mass index (BMI), metabolic syndrome (MetS), blood lipids and glucose levels. Random effects models were used for meta-analyses, and effects reported as risk difference (RD) or mean difference (MD) and their 95\% confidence interval (CI). Subgroup analyses by time of follow up, publication year, and confounder adjustment were performed.

Results: We evaluated 41 cohorts including $3300 \mathrm{PE} / \mathrm{E}$ and 13,967 normotensive controls. Women were followed up from 3 months after delivery up to 32 years postpartum. In comparison to controls, PE/E significantly increased systolic $\mathrm{BP}$ ( $\mathrm{MD}=8.3 \mathrm{mmHg}, 95 \% \mathrm{CI} 6.8$ to 9.7 ), diastolic $\mathrm{BP}(\mathrm{MD}=6.8 \mathrm{mmHg}, 95 \% \mathrm{CI} 5.6$ to 8.0 ), $\mathrm{BMI}$ ( $\mathrm{MD}=$ $2.0 \mathrm{~kg} / \mathrm{m}^{2} ; 95 \% \mathrm{CI} 1.6$ to 2.4 ), waist ( $\mathrm{MD}=4.3 \mathrm{~cm}, 95 \% \mathrm{CI} 3.1$ to 5.5 ), waist-to-hip ratio (MD $=0.02,95 \% \mathrm{CI} 0.01$ to 0.03 ), weight ( $\mathrm{MD}=5.1 \mathrm{~kg}, 95 \% \mathrm{Cl} 2.2$ to 7.9 ), total cholesterol ( $\mathrm{MD}=4.6 \mathrm{mg} / \mathrm{dL}, \mathrm{Cl} 1.5$ to 7.7$), \mathrm{LDL}(\mathrm{MD}=$ $4.6 \mathrm{mg} / \mathrm{dL} ; 95 \% \mathrm{Cl} 0.2$ to 8.9 ), triglycerides ( $\mathrm{MD}=7.7 \mathrm{mg} / \mathrm{dL}, 95 \% \mathrm{Cl} 3.6$ to 11.7 ), glucose ( $\mathrm{MD}=2.6 \mathrm{mg} / \mathrm{dL}, 95 \%$ CI 1.2 to 4.0 ), insulin ( $\mathrm{MD}=19.1 \mathrm{pmol} / \mathrm{L}, 95 \% \mathrm{CI} 11.9$ to 26.2 ), HOMA-IR index ( $\mathrm{MD}=0.7,95 \% \mathrm{CI} 0.2$ to 1.2 ), $\mathrm{C}$ reactive protein ( $\mathrm{MD}=0.05 \mathrm{mg} / \mathrm{dL}, 95 \% \mathrm{Cl} 0.01$ to 0.09 ), and the risks of hypertension ( $\mathrm{RD}=0.24,95 \% \mathrm{Cl} 0.15$ to 0.33 ) and MetS ( $R D=0.11,95 \% \mathrm{Cl} 0.08$ to 0.15 ). Also, $\mathrm{PE} / \mathrm{E}$ reduced HDL levels ( $\mathrm{MD}=-2.15 \mathrm{mg} / \mathrm{dL}, 95 \% \mathrm{Cl}-$ 3.46 to -0.85 ). Heterogeneity of effects was high for most outcomes. Risk of bias was moderate across studies. Subgroup analyses showed similar effects as main analyses.

Conclusion: Women who had PE/E have worse metabolic and biochemical profile than those without PE/E in an intermediate to long term follow up period.
\end{abstract}

(c) 2019 Elsevier Inc. All rights reserved.

Abbreviations: ACOG, American College of Obstetrics and Gynecologists; ASSHPC, Australasian Society for the Study of Hypertension in Pregnancy Consensus; BMI, body mass index;

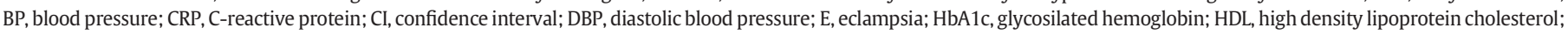

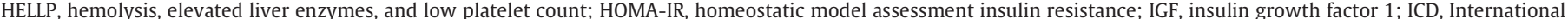

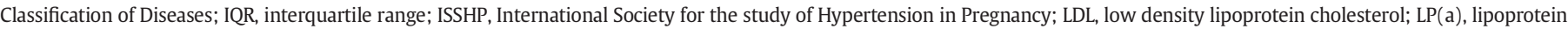

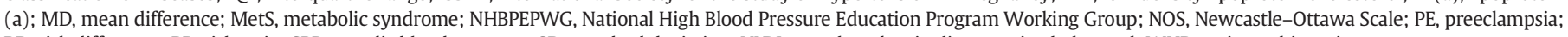
RD, risk difference; RR, risk ratio; SBP, systolic blood pressure; SD, standard deviation; VLDL, very low density lipoprotein cholesterol; WHR, waist-to-hip ratio.

* Corresponding author at: Department of Obstetrics and Gynecology, Facultad de Medicina, Universidad de Zaragoza, Domingo Miral s/n, Zaragoza 50009, Spain.

E-mail addresses: vaventura.92@gmail.com (V. Alonso-Ventura), Yangzhou.li@uconn.edu (Y. Li), lepiscean@gmail.com (V. Pasupuleti), yuani.roman-morillo@hhchealth.org (Y.M. Roman), adrian.hernandez-diaz@uconn.edu (A.V. Hernandez), faustino.perez@unizar.es (F.R. Pérez-López). 


\section{Introduction}

Hypertensive disorders of pregnancy (HDP) are a heterogeneous group of syndromes affecting $3-10 \%$ of pregnancies, and include preeclampsia (PE), eclampsia (E), gestational hypertension, and pre-gestational hypertension [1,2]. PE and $\mathrm{E}$ have as a common definition the presence of new onset hypertension and proteinuria diagnosed during the second half ( $>20$ weeks) of pregnancy. E is associated with tonic-clonic seizures and general complications in a woman with or without preeclampsia. Hemolysis, elevated liver enzymes, and low platelet count (HELLP) syndrome is a rare complication of PE/E which may be accompanied of fatigue, edema, headache, nausea, abdominal pain, visual alterations, hemorrhage, intravascular coagulation, kidney failure and placental abruption [1,2].

$\mathrm{PE} / \mathrm{E}$ have negative consequences on maternal and fetal health during pregnancy, including increased perinatal mortality, preterm births, small for gestational age infants, high rate of cesarean deliveries, and other adverse outcomes even at later postnatal periods [3-6]. PE/E are associated with elevated blood pressure, inflammation and endothelial dysfunction, and these findings may remain after delivery and contribute to future maternal cardiovascular risk [7-11]. Furthermore, two recent meta-analyses reported that PE was independently associated with higher risk of future diabetes and cardiovascular events [12,13]. In particular, PE increased the risk of future diabetes (risk ratio [RR] 2.37, 95\% confidence interval $[\mathrm{CI}] 1.89,2.97$ ) appearing in women as early as during 1 year postpartum (RR $1.97,95 \% \mathrm{CI} 1.35,2.87$ ) and persisting the risk up to 10 years after delivery (RR $1.95,95 \% \mathrm{CI}$ $1.28,2.97$ ) [12]. PE was also independently associated with higher risk of future heart failure (RR 4.19, 95\% CI 2.09-8.38), coronary heart disease (RR 2.50, 95\% CI 1.43-4.37), cardiovascular disease death (RR 2.21, 95\% CI 1.83-2.66), and stroke (RR 1.81, 95\% CI 1.29-2.55) [13]. Risks persisted after different confounder adjustments.
We systematically evaluated the association between PE/E and metabolic and biochemical outcomes from observational studies with intermediate and long term of follow up.

\section{Methods}

This systematic review was reported according to the MetaAnalysis Of Observational Studies in Epidemiology (MOOSE) Group guidelines [14]. Formal institutional review board approval was not required as this manuscript only addresses data extracted from already published studies.

\subsection{Study search}

PubMed-Medline, Scopus, Web of Science, Cochrane Library, and EMBASE were searched from inception to November 2018 for observational studies evaluating the association between PE/E and metabolic and biochemical outcomes after delivery. Studies were included irrespective of age, parity, ethnicity, country of origin, publication date and language. A search strategy was developed for PubMed, and modified accordingly for other databases. Also, reference lists from selected studies were hand searched. Keywords were preeclampsia, eclampsia, HELLP, and each metabolic and biochemical outcome. The full Pubmed search strategy using Boolean operators AND or OR can be found in Appendix A, Supplementary Table 1.

PE, E and HELLP syndrome have a common definition: new onset hypertension and proteinuria appearing after 20 weeks of pregnancy according to different scientific societies such as the American College of Obstetrics and Gynecologists (ACOG) [2], the International Society for the study of Hypertension in Pregnancy (ISSHP) [15], the National High Blood Pressure Education Program Working Group (NHBPEPWG) [16], the World Health Organization International Classification of Diseases (ICD) [17], or the Australasian Society for the Study of Hypertension in Pregnancy Consensus

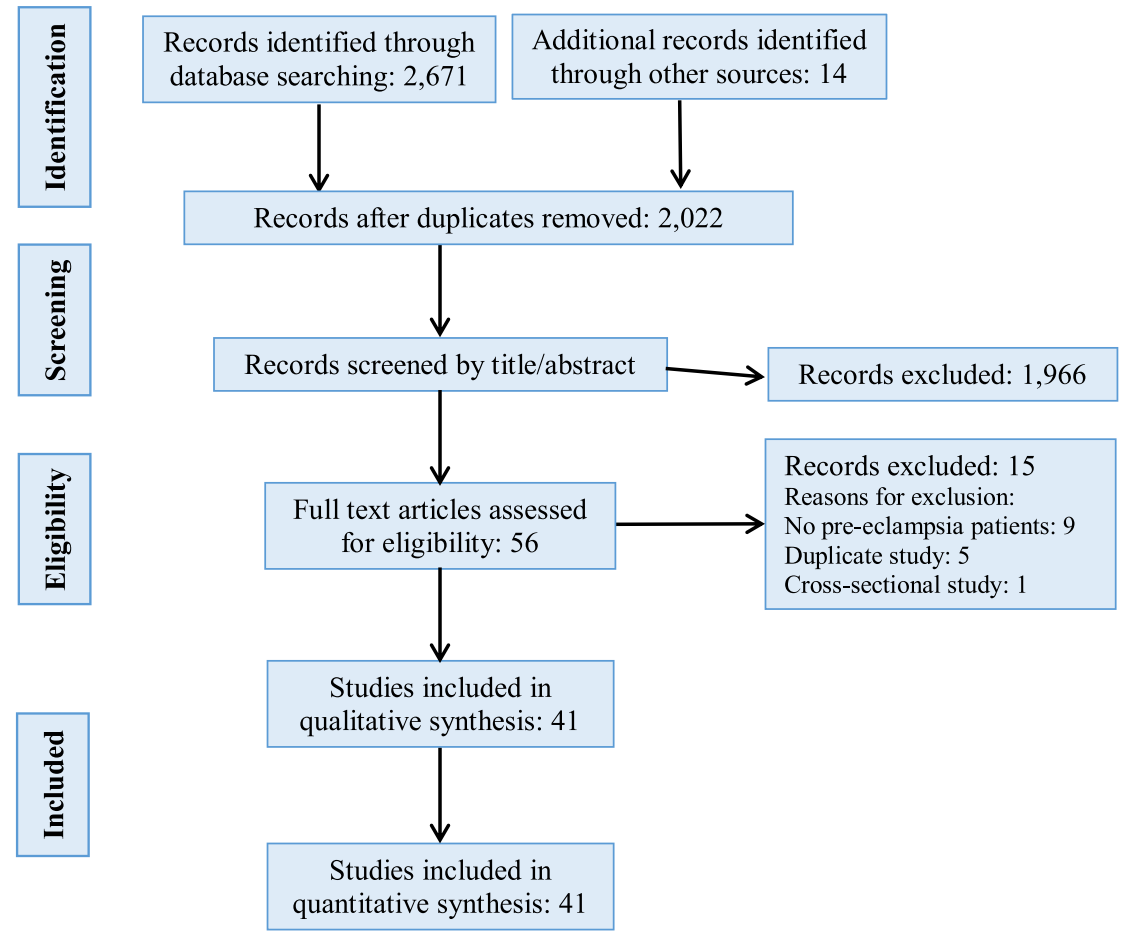

Fig. 1. Flow diagram of included studies. 


\begin{tabular}{|c|c|c|c|c|c|c|c|c|}
\hline \multirow[b]{2}{*}{$\begin{array}{l}\text { Author, year } \\
\text { [reference] }\end{array}$} & \multirow[b]{2}{*}{ Country; study design } & \multirow[b]{2}{*}{ Exclusion reasons } & \multicolumn{2}{|c|}{ Data during the index pregnancy: preeclampsia (PE) } & \multicolumn{2}{|c|}{ Data during the index pregnancy: control (CG) } & \multirow[b]{2}{*}{$\begin{array}{l}\text { Time of follow up } \\
\text { and/or age }\end{array}$} & \multirow[b]{2}{*}{$\begin{array}{l}\text { Matching variables } \\
\text { for controls }\end{array}$} \\
\hline & & & $\begin{array}{l}\text { Inclusion criteria; } \mathrm{n} \\
\text { (women); age at pregnancy }\end{array}$ & $\begin{array}{l}\text { Parity index } \\
\text { pregnancy; GAD, } \\
\text { weeks }\end{array}$ & $\begin{array}{l}\text { CG definition; } \mathrm{n} \text { (women); } \\
\text { age at pregnancy }\end{array}$ & $\begin{array}{l}\text { Parity index } \\
\text { pregnancy. GAD, } \\
\text { weeks }\end{array}$ & & \\
\hline $\begin{array}{l}\text { Christensen M, } \\
2016 \text { [33] }\end{array}$ & $\begin{array}{l}\text { Denmark; Retrospective } \\
\text { cohort study. }\end{array}$ & $\begin{array}{l}\text { Unexposed women who } \\
\text { experienced hypertensive } \\
\text { disorder of pregnancy before } \\
\text { or after 2001-2004 were } \\
\text { excluded. }\end{array}$ & $\begin{array}{l}\text { PE according to the ACOG. } \\
\mathrm{N}=21 \text { patients with PE in } \\
\text { 2001-2004; Age: time since } \\
\text { delivery, years: } 10.28 \pm \\
0.70 \text { years }\end{array}$ & $\begin{array}{l}\text { Primiparous } n=16 \\
\text { (76\%); GAD: } 262 \pm \\
19 \text { days }\end{array}$ & $\begin{array}{l}\mathrm{N}=21 \text { normotensive } \\
\text { pregnant women without PE, } \\
\text { in 2001-2004; Age: time } \\
\text { since delivery, years: } 10.27 \pm \\
0.51 \text {. years }\end{array}$ & $\begin{array}{l}\text { Primiparous } \mathrm{n}=10 \\
\text { (48\%); GAD: } 280 \pm \\
9 \text { days }\end{array}$ & $\begin{array}{l}\text { Ten year after delivery. } \\
\text { Age: exposed women } \\
40.75 \pm 2.7 \text { years; } \\
\text { non-exposed women } \\
40.67 \pm 2.3 \text { years }\end{array}$ & $\begin{array}{l}\text { Age ( } \pm 2 \text { years) and } \\
\text { time since delivery } \\
\text { ( } \pm 1 \text { year) }\end{array}$ \\
\hline $\begin{array}{l}\text { Coffeng SM, } 2011 \\
\text { [34] }\end{array}$ & $\begin{array}{l}\text { The Netherlands; } \\
\text { Prospective cohort } \\
\text { study }\end{array}$ & $\begin{array}{l}\text { Five cases and four controls } \\
\text { were excluded because of } \\
\text { current pregnancy or } \\
\text { breastfeeding. One case was } \\
\text { excluded because of a history } \\
\text { of breast cancer with } \\
\text { chemotherapy. }\end{array}$ & $\begin{array}{l}\text { Severe early-onset PE } \\
\text { according to the ISSHP; } \mathrm{N}= \\
16 \text { severe early-onset PE; } \\
\text { Age: } 29.7 \pm 4.8 \text { years }\end{array}$ & $\begin{array}{l}\text { Parity and GAD not } \\
\text { reported }\end{array}$ & $\begin{array}{l}\mathrm{N}=17 \text { women with } \\
\text { uncomplicated pregnancies; } \\
\text { Age: } 30.7 \pm 3.6 \text { years }\end{array}$ & $\begin{array}{l}\text { Parity and GAD not } \\
\text { reported }\end{array}$ & $\begin{array}{l}\text { Study } 4 \text { years after } \\
\text { index delivery. }\end{array}$ & Not matched \\
\hline $\begin{array}{l}\text { Dantas EMM, } 2013 \\
\text { [35] }\end{array}$ & $\begin{array}{l}\text { Brazil; Prospective } \\
\text { cohort study }\end{array}$ & Not reported & $\begin{array}{l}\text { PE according to the diagnostic } \\
\text { criteria of the NHBPEPWG, } \\
\mathrm{N}=10 \text { women (one women } \\
\text { developed HELLP syndrome); } \\
\text { Age: } 27.0 \pm 6.7 \text { years }\end{array}$ & $\begin{array}{l}\text { Parity: median } 0.5 \\
\text { [half interquartile } \\
\text { 1.0]; GAD: } 37.6 \pm \\
3.2 \text { weeks }\end{array}$ & $\begin{array}{l}\text { Normotensive pregnancies. } \\
\mathrm{N}=17 \text { women; Age: } 26.0 \pm \\
2.5 \text { years }\end{array}$ & $\begin{array}{l}\text { Parity: Median } 1.0 \\
\text { [half interquartile } \\
\text { 1.0]; GAD: } 39.1 \pm \\
\text { 1.9 weeks }\end{array}$ & $\begin{array}{l}\text { Study at } 5 \text { years } \\
\text { follow-up. }\end{array}$ & Not matched \\
\hline Drost JT, 2012 [36] & $\begin{array}{l}\text { The Netherlands; Pro- } \\
\text { spective cohort study }\end{array}$ & Pregnant or lactating women & $\begin{array}{l}\mathrm{PE}<32 \text { weeks. } \mathrm{N}=339 \\
\text { women with PE according } \\
\text { to the ISSHP; Age: } 29,8 \pm \\
3,8 \text { years }\end{array}$ & $\begin{array}{l}\text { Number of } \\
\text { pregnancies: } 2.7 \pm \\
\text { 1.4; GAD: not } \\
\text { reported }\end{array}$ & $\begin{array}{l}\text { Pregnant women without } \mathrm{PE} \text {, } \\
\mathrm{N}=332 \text { women without } \mathrm{PE} \\
\text { Age: } 28,6 \pm 4,1 \text { years }\end{array}$ & $\begin{array}{l}\text { Number of } \\
\text { pregnancies: } 3.1 \pm \\
\text { 1.4; GAD: not } \\
\text { reported }\end{array}$ & $\begin{array}{l}\text { Study } 10 \text { years post } \\
\text { index-delivery. }\end{array}$ & Age \\
\hline Forest JC, 2005 [37] & $\begin{array}{l}\text { Canada; Retrospective } \\
\text { cohort study }\end{array}$ & $\begin{array}{l}\text { Pregnant women and women } \\
\text { who had delivered within } 6 \\
\text { months of the scheduled visit }\end{array}$ & $\begin{array}{l}\text { PE according to the ACOG; } \\
N=63 \text { PE; Age: } 27.4 \pm \\
3.9 \text { years }\end{array}$ & $\begin{array}{l}\text { Parity: not reported } \\
\text { GAD: } 38.3 \pm 2.6 \\
\text { weeks }\end{array}$ & $\begin{array}{l}\text { Pregnant women without PE. } \\
\mathrm{N}=168 \text { controls matched for } \\
\text { age and year of index } \\
\text { delivery; Age } 27.0 \pm 4.2 \text { years }\end{array}$ & $\begin{array}{l}\text { Parity not reported; } \\
\text { GAD: } 39.4 \pm 2.1 \\
\text { weeks }\end{array}$ & $\begin{array}{l}\text { Average period from } \\
\text { the index pregnancy to } \\
\text { the scheduled study } \\
7.8 \text { years (range } \\
5.1-13.0 \text { years). }\end{array}$ & $\begin{array}{l}\text { Maternal age and } \\
\text { year of delivery of } \\
\text { the index pregnancy } \\
\text { (within } 1 \text { year). }\end{array}$ \\
\hline $\begin{array}{l}\text { Freeman DJ, } 2004 \\
\text { [38] }\end{array}$ & $\begin{array}{l}\text { United Kingdom; } \\
\text { Retrospective cohort } \\
\text { study }\end{array}$ & $\begin{array}{l}\text { Other hypertensive disorders } \\
\text { of pregnancy }\end{array}$ & $\begin{array}{l}\text { PE according to the ISSHP, } \\
\mathrm{N}=40 \text { pregnancies } \\
\text { between } 1975 \text { and } 1985 \\
\text { Age: } 24.9 \pm 5.2 \text { years. }\end{array}$ & $\begin{array}{l}\text { Primiparous and } \\
\text { multiparous women; } \\
\text { GAD: } 35.3 \pm 3.8 \\
\text { weeks }\end{array}$ & $\begin{array}{l}\text { Women without PE, } \mathrm{N}=38 \\
\text { pregnancies; Age: } 24.7 \pm 3.9 \\
\text { years. }\end{array}$ & $\begin{array}{l}38 \text { women without } \\
\text { adverse outcomes; } \\
\text { GAD: } 39.1 \pm 2.8 \\
\text { weeks }\end{array}$ & $\begin{array}{l}\text { Endpoints studied } 20 \\
\text { years after } \\
\text { pregnancies }\end{array}$ & $\begin{array}{l}\text { Age- and } \\
\text { parity-matched } \\
\text { controls between } \\
1975 \text { and } 1985\end{array}$ \\
\hline $\begin{array}{l}\text { Garovic VD, } 2017 \\
\text { [39] }\end{array}$ & $\begin{array}{l}\text { United States; } \\
\text { Retrospective cohort } \\
\text { study }\end{array}$ & $\begin{array}{l}\text { Women with previous CVD } \\
\text { events, such as myocardial } \\
\text { infarction, congestive heart } \\
\text { failure, stroke, and dysrhythmias }\end{array}$ & $\begin{array}{l}\text { PE: } 40 \text { women with } \\
\text { preeclampsia according to } \\
\text { the ICD } 9 \text { codes, who } \\
\text { delivered between } 1976 \text { and } \\
\text { 1982; Age: } 24.2 \pm 3.7\end{array}$ & $\begin{array}{l}\text { Parity: not reported; } \\
\text { GAD: not reported }\end{array}$ & $\begin{array}{l}40 \text { age- and parity-matched } \\
\text { normotensive women, who } \\
\text { delivered between } 1976 \text { and } \\
\text { 1982; Age: } 24.3 \pm 3.4 \text { years }\end{array}$ & $\begin{array}{l}\text { Parity: parity } \\
\text { matched; GAD: not } \\
\text { reported }\end{array}$ & $\begin{array}{l}\text { Women who delivered } \\
\text { in 1976-1982, were } \\
\text { studied in 2014-2015; } \\
\text { Age: PE } 59.4 \pm 4.8 \text {; } \\
\text { control: } 59.7 \pm 4.5 \text { years }\end{array}$ & Age and parity \\
\hline $\begin{array}{l}\text { Girouard D J, } 2007 \\
{[40]}\end{array}$ & $\begin{array}{l}\text { Canada; Retrospective } \\
\text { cohort study }\end{array}$ & $\begin{array}{l}\text { Multiparous women and } \\
\text { women with known renal } \\
\text { diseases, diabetes mellitus, } \\
\text { or CVD }\end{array}$ & $\begin{array}{l}\text { PE: } 63 \text { women according to } \\
\text { the diagnostic criteria of the } \\
\text { NHBPEPWG; Age: } 27.4 \pm \\
3.9 \text { years }\end{array}$ & $\begin{array}{l}\text { Parity: not reported; } \\
\text { GAD: } 38.3 \pm 2.6\end{array}$ & $\begin{array}{l}\mathrm{N}=168 \text { women with } \\
\text { normotensive pregnancy. } \\
\text { Mean age: } 27.0 \pm 4.2 \text { years }\end{array}$ & $\begin{array}{l}\text { Parity: not reported; } \\
\text { GAD: } 39.4_{-} \pm 2.1\end{array}$ & $\begin{array}{l}\text { Women were studied } \\
7.8 \text { years after their } \\
\text { first delivery. Case } \\
\text { women were more } \\
\text { overweight compared } \\
\text { with controls }\end{array}$ & $\begin{array}{l}\text { Maternal age and } \\
\text { year of delivery of } \\
\text { the index pregnancy } \\
\text { (within } 1 \text { year) }\end{array}$ \\
\hline $\begin{array}{l}\text { Hamad RR, } 2007 \\
\text { [41] }\end{array}$ & $\begin{array}{l}\text { Sweden; Retrospective } \\
\text { cohort study }\end{array}$ & $\begin{array}{l}\text { No hormonal therapy for } 6 \\
\text { months before the study or } \\
\text { other drug treatment; } \\
\text { breast-feeding terminated. } \\
\text { No DM, gestational DM, } \\
\text { coagulation disorders, renal } \\
\text { diseases, and chronic } \\
\text { hypertension }\end{array}$ & $\begin{array}{l}\text { Severe } P E \text { according to the } \\
\text { ISSHP, } N=18 \text { women; Age: } \\
30 \pm 4 \text { years }\end{array}$ & $\begin{array}{l}\text { Parity: one; GAD: } \\
\text { not reported }\end{array}$ & $\begin{array}{l}\mathrm{N}=17 \text { age-matched controls; } \\
\text { Mean age: } 31 \pm 4 \text { years }\end{array}$ & $\begin{array}{l}\text { Parity: one; GAD: } \\
\text { not reported }\end{array}$ & $\begin{array}{l}\text { Women were studied } \\
7.8 \text { years after delivery }\end{array}$ & Age and parity \\
\hline He S, 1999 [42] & $\begin{array}{l}\text { Sweden; Retrospective } \\
\text { cohort study }\end{array}$ & $\begin{array}{l}\text { Negative history of } \\
\text { hematological, cardiovascular, } \\
\text { hepatic or renal disorder } \\
\text { before index pregnancy }\end{array}$ & $\begin{array}{l}\text { PE } 25 \text { women ( } 11 \text { mild PE, } \\
\text { and } 14 \text { severe PE); Age: } 33 \pm \\
6 \text { years }\end{array}$ & $\begin{array}{l}\text { Parity: } 32 \text { primipara; } \\
\text { GAD: not reported }\end{array}$ & $\begin{array}{l}\mathrm{N}=24 \text { women; Age: } \\
\text { Matched by age, parity and } \\
\text { index of pregnancy } 34 \pm 6 \\
\text { years }\end{array}$ & $\begin{array}{l}\text { Parity: } 29 \\
\text { primiparous; GAD: } \\
\text { not reported }\end{array}$ & $\begin{array}{l}\text { Women were examined } \\
2-5(4.5 . \pm 0.8) \text { years } \\
\text { after delivery }\end{array}$ & $\begin{array}{l}\text { Age, parity at index } \\
\text { pregnancy and time } \\
\text { of delivery }\end{array}$ \\
\hline
\end{tabular}




\begin{tabular}{|c|c|c|c|c|c|c|c|c|}
\hline $\begin{array}{l}\text { Hubel CA, } 2008 \\
\text { [43] }\end{array}$ & $\begin{array}{l}\text { Iceland; Retrospective } \\
\text { cohort study }\end{array}$ & $\begin{array}{l}\text { Women with hypertension } \\
\text { before week } 20 \text { of gestation or } \\
\text { reported history of } \\
\text { hypertension were excluded. } \\
\text { None of the women had a } \\
\text { history of gestational DM }\end{array}$ & $\begin{array}{l}\text { Eclampsia: } 25 \text { women; Age: } \\
\text { not reported }\end{array}$ & $\begin{array}{l}\text { Parity: not reported; } \\
\text { GAD: not reported }\end{array}$ & $\begin{array}{l}\text { Control } 28 \text { women with } \\
\text { uncomplicated pregnancy; } \\
\text { Age not reported }\end{array}$ & $\begin{array}{l}\text { Parity: not reported; } \\
\text { GAD: not reported }\end{array}$ & $\begin{array}{l}\text { Deliveries between } \\
1931 \text { and } 1996 \text {. Women } \\
\text { were } 50 \text { to } 67 \text { years old } \\
\text { at reexamination } \\
\text { ( } 32 \text { years after delivery) }\end{array}$ & $\begin{array}{l}\text { Age, age at } \\
\text { pregnancy, and parity }\end{array}$ \\
\hline Innes KE, 2005 [44] & $\begin{array}{l}\text { United States; } \\
\text { Retrospective cohort } \\
\text { study }\end{array}$ & $\begin{array}{l}\text { Cancer, hypertension, renal } \\
\text { disease, or diabetes. None } \\
\text { were currently breast-feeding. } \\
\text { A history of infertility, } \\
\text { multi-fetal gestation, or } \\
\text { gestational DM currently on } \\
\text { medications known to alter } \\
\text { hormone or lipid levels }\end{array}$ & $\begin{array}{l}\text { Cases: } 13 \text { women with PE } \\
\text { according to the ACOG in } \\
\text { their first pregnancies; Age: } \\
33.9 \pm 0.9 \text { years }\end{array}$ & $\begin{array}{l}\text { Parity: primiparous; } \\
\text { GAD: not reported }\end{array}$ & $\begin{array}{l}13 \text { pregnant women matched } \\
\text { to cases on race/ethnicity, } \\
\text { current age, and age at } \\
\text { delivery. Age: } 33.3 \pm 0.9 \\
\text { years }\end{array}$ & $\begin{array}{l}\text { Parity: Primiparous; } \\
\text { GAD: not reported }\end{array}$ & $\begin{array}{l}\text { All subjects were } \\
\text { menstruating regularly } \\
\text { at the time of the } \\
\text { study. Follow up } \\
\text { interval ranged from } \\
1 \text { to } 10 \text { years and } \\
\text { averaged } 3.69 \pm 0.47 \\
\text { years }\end{array}$ & $\begin{array}{l}\text { Cases on } \\
\text { race/ethnicity, } \\
\text { current age, and age } \\
\text { at delivery }\end{array}$ \\
\hline $\begin{array}{l}\text { Kvehaugen AS, } \\
2010[45]\end{array}$ & $\begin{array}{l}\text { Norway; Retrospective } \\
\text { cohort study }\end{array}$ & $\begin{array}{l}\text { Women with current } \\
\text { pregnancy or lactation were } \\
\text { excluded. None of the women } \\
\text { had CVD and none were } \\
\text { diagnosed with de novo DM2 } \\
\text { after index pregnancy }\end{array}$ & $\begin{array}{l}\text { PE: } 23 \text { women with } \\
\text { preeeclampsia; Age: } 30.0 \\
{[27.0,32.0]}\end{array}$ & $\begin{array}{l}\text { Parity: } 2.0[1.0,2.0] \text {, } \\
\text { Multiparous } 60 \% \text {; } \\
\text { GAD: Median } 32.3 \\
\text { weeks }\end{array}$ & $\begin{array}{l}15 \text { control matched } \\
\text { normotensive pregnancy at the } \\
\text { same hospital; Age: } 34.0 \\
{[32.0,37.0]}\end{array}$ & $\begin{array}{l}\text { Parity: } 2.0[2.0,3.0] \text {, } \\
\text { Multiparous } 30 \% \text {; } \\
\text { GAD: Median } 38.6 \\
\text { weeks }\end{array}$ & $\begin{array}{l}\text { Age at the study: PE } \\
\text { women } 36.0 \text { [33.0, } \\
\text { 39.0]; Controls: } 40.5 \\
{[39.0,44.0]}\end{array}$ & Not matched \\
\hline $\begin{array}{l}\text { Laivuori H, } 1996 \\
\text { [46] }\end{array}$ & $\begin{array}{l}\text { Finland; Retrospective } \\
\text { cohort study }\end{array}$ & $\begin{array}{l}\text { Women with hysterectomy; } \\
\text { use of levonorgestrel-release } \\
\text { intrauterine device, } \\
\text { hormone replacement } \\
\text { therapy, or progestin-only } \\
\text { contraception }\end{array}$ & $\begin{array}{l}\text { PE or eclampsia in } 22 \text { women; } \\
\text { Age: } 24.8 \pm 20.9 \text { years }\end{array}$ & $\begin{array}{l}\text { Parity: First } \\
\text { pregnancy; GAD: } \\
36.2 \pm 0.5 \text { weeks }\end{array}$ & $\begin{array}{l}\text { Control women: } 22 ; \\
\text { Age: } 25.0 \pm 0.9 \text { years }\end{array}$ & $\begin{array}{l}\text { Parity: First } \\
\text { pregnancy; GAD: } \\
40.1 \pm 0.4 \text { weeks }\end{array}$ & $\begin{array}{l}\text { Years since delivery: } \\
\text { PE-eclampsia group: } \\
41.8 \pm 0.9 \text { years; } \\
\text { Control group: } 41.8 \pm \\
0.9 \text { years }\end{array}$ & Age \\
\hline $\begin{array}{l}\text { Lampinen KH, } 2008 \\
\text { [47] }\end{array}$ & $\begin{array}{l}\text { Finland; Retrospective } \\
\text { cohort study }\end{array}$ & $\begin{array}{l}\text { Women with concomitant } \\
\text { disease, such as DM or a } \\
\text { history of gestational DM, } \\
\text { chronic hypertension, and } \\
\text { kidney disease or coagulation } \\
\text { disorders were excluded }\end{array}$ & $\begin{array}{l}\text { Severe PE: } 28 \text { non-obese } \\
\text { women with previous severe } \\
\text { preeclampsia or eclampsia; } \\
\text { Age: } 33 \pm 5 \text { years }\end{array}$ & $\begin{array}{l}\text { Primiparity: } 14 / 28 \\
\text { (50\%); GAD: } 33 \text { [29, } \\
\text { 36] weeks }\end{array}$ & $\begin{array}{l}\text { Control women: } 20 \text { women } \\
\text { with a previous normotensive } \\
\text { pregnancy; Age } 30 \pm 4\end{array}$ & $\begin{array}{l}\text { Primiparity: } 3 / 20 \\
\text { (15\%); GAD: } 40 \text { ([40 } \\
\text { to 41]: }\end{array}$ & $\begin{array}{l}\text { Women were studied } \\
5 \text { to } 6 \text { years after the } \\
\text { index pregnancy. }\end{array}$ & Not matched \\
\hline $\begin{array}{l}\text { Mangos GJ, } 2012 \\
\text { [48] }\end{array}$ & $\begin{array}{l}\text { Australia; Retrospective } \\
\text { cohort study }\end{array}$ & $\begin{array}{l}\text { Pregnant women were } \\
\text { excluded who had DM, } \\
\text { prior essential hypertension } \\
\text { in pregnancy or renal } \\
\text { disease }\end{array}$ & $\begin{array}{l}\text { PE: } 39 \text { women according to the } \\
\text { ASSHPC Statement criteria for } \\
\text { the diagnosis preeclampsia; } \\
\text { Age: } 37 \pm 6 \text { years }\end{array}$ & $\begin{array}{l}\text { Multiparous: } 26 \\
\text { (67\%); GAD: not } \\
\text { reported }\end{array}$ & $\begin{array}{l}\text { Control group: } 35 \text { women; } \\
\text { Age: } 38 \pm 6 \text { years }\end{array}$ & $\begin{array}{l}\text { Multiparous women } \\
30 \text { (86\%); GAD: not } \\
\text { reported }\end{array}$ & $\begin{array}{l}\text { Years postpartum: PE } \\
\text { group: } 3.8 \text { (2.5-5.0); } \\
\text { control group: } 4.3 \\
(2.8-7.0)\end{array}$ & BMI \\
\hline $\begin{array}{l}\text { Manten GTR, } 2007 \\
\text { [49] }\end{array}$ & $\begin{array}{l}\text { The Netherlands; } \\
\text { Retrospective cohort } \\
\text { study }\end{array}$ & $\begin{array}{l}\text { Women with fasting glucose } \\
\text { levels } \geq 7.0 \mathrm{mmol} / \mathrm{L}\end{array}$ & $\begin{array}{l}\text { PE: } 256 \text { women with } \\
\text { preeclampsia according to } \\
\text { the ACOG criteria, HELLP } \\
\text { syndrome } n=163(64 \%) ; \\
\text { Age: } 31 \pm 4\end{array}$ & $\begin{array}{l}\text { Primiparous } 203 \\
\text { (79\%); GAD: } 217 \pm \\
28 \text { days }\end{array}$ & $\begin{array}{l}\text { Control group: } 53 \text { women; } \\
\text { Age: } 33 \pm 4\end{array}$ & $\begin{array}{l}\text { Primiparous } 31 \\
\text { (58\%); GAD: } 283 \pm \\
10 \text { days }\end{array}$ & $\begin{array}{l}\text { Women were studied } \\
\text { at least } 3 \text { months after } \\
\text { delivery, and after } \\
\text { ending lactation }\end{array}$ & \\
\hline $\begin{array}{l}\text { McDonald SD, } 2013 \\
\text { [50] }\end{array}$ & $\begin{array}{l}\text { Canada; Retrospective } \\
\text { cohort study }\end{array}$ & $\begin{array}{l}\text { Women with prior chronic } \\
\text { hypertension in pregnancy; } \\
\text { gestational hypertension; } \\
\text { known CVD; chronic medical } \\
\text { conditions such as liver } \\
\text { disease, untreated hyper or } \\
\text { hypothyroidism, renal } \\
\text { disease, or malignancy }\end{array}$ & $\begin{array}{l}\text { PE: } 109 \text { women with } \\
\text { preeclampsia according to } \\
\text { the NHBPEPW group; Age of } \\
\text { oldest child median } 19 \text { [ } 15, \\
25] \text { years; } \\
\text { Age at index pregnancy: not } \\
\text { reported }\end{array}$ & $\begin{array}{l}\text { Parity not reported; } \\
\text { GAD not reported }\end{array}$ & $\begin{array}{l}\text { Control group: } 219 \text { women } \\
\text { without PE; Age of oldest child, } \\
\text { median } 21 \text { [16, 28]; Age at } \\
\text { index pregnancy: not reported }\end{array}$ & $\begin{array}{l}\text { Parity not reported; } \\
\text { GAD not reported }\end{array}$ & $\begin{array}{l}\text { Study performed two } \\
\text { decades after delivery; } \\
\text { Age: PE group } 49 \text { ([QR } \\
\text { 44-55] years; Control } \\
\text { group: } 49 \text { [IQR 45-56] } \\
\text { years }\end{array}$ & $\begin{array}{l}\text { Maternal age } \pm 3 \\
\text { years and child's age } \\
\pm 5 \text { years }\end{array}$ \\
\hline Nisell H, 1999 [51] & $\begin{array}{l}\text { Sweden; Retrospective } \\
\text { cohort study }\end{array}$ & $\begin{array}{l}\text { History of CVD, renal or } \\
\text { endocrine disease. None had } \\
\text { a diagnosis of gestational } \\
\text { DM. None were taking any } \\
\text { drugs or any form of } \\
\text { hormonal contraception. } \\
\text { Breast feeding was completed } \\
\text { in all cases }\end{array}$ & $\begin{array}{l}\text { PE: } 21 \text { women with } \\
\text { preeclampsia according to } \\
\text { the ACOG criteria; Age: } 30 \\
\text { SEM } 1\end{array}$ & $\begin{array}{l}\text { Parity: Primiparous } \\
\text { 14; GAD: } 37.1 \text { SEM } \\
0.8\end{array}$ & $\begin{array}{l}\text { Control group; } 22 \text { women; } \\
\text { Age: } 30 \text { SEM } 1\end{array}$ & $\begin{array}{l}\text { Parity: Primiparous } \\
\text { 9; GAD: 40.1 SEM } \\
0.3\end{array}$ & $\begin{array}{l}\text { Women were followed } \\
\text { up to } 26-119 \text { weeks } \\
\text { after delivery }\end{array}$ & $\begin{array}{l}\text { Age, pregnancy } \\
\text { during } 1995\end{array}$ \\
\hline Nohira T, 2013 [52] & Japan; Retrospective & Patients who had CVD prior to & PE: 58 women with severe & Parity: $0.896 \pm$ & 61 normal pregnancies; Age: & Parity: $0.874 \pm$ & Elapse time from & ge, parity, \\
\hline
\end{tabular}


Table 1 (continued)

\begin{tabular}{|c|c|c|c|c|c|c|c|c|}
\hline \multirow[b]{2}{*}{$\begin{array}{l}\text { Author, year } \\
\text { [reference] }\end{array}$} & \multirow[b]{2}{*}{ Country; study design } & \multirow[b]{2}{*}{ Exclusion reasons } & \multicolumn{2}{|c|}{ Data during the index pregnancy: preeclampsia (PE) } & \multicolumn{2}{|c|}{ Data during the index pregnancy: control (CG) } & \multirow[b]{2}{*}{$\begin{array}{l}\text { Time of follow up } \\
\text { and/or age }\end{array}$} & \multirow[b]{2}{*}{$\begin{array}{l}\text { Matching variables } \\
\text { for controls }\end{array}$} \\
\hline & & & $\begin{array}{l}\text { Inclusion criteria; } \mathrm{n} \\
\text { (women); age at pregnancy }\end{array}$ & $\begin{array}{l}\text { Parity index } \\
\text { pregnancy; GAD, } \\
\text { weeks }\end{array}$ & $\begin{array}{l}\text { CG definition; } n \text { (women); } \\
\text { age at pregnancy }\end{array}$ & $\begin{array}{l}\text { Parity index } \\
\text { pregnancy. GAD, } \\
\text { weeks }\end{array}$ & & \\
\hline & cohort study & pregnancy were excluded. & $\begin{array}{l}\text { preeclampsia; Age: } 27.37 \pm \\
3.44 \text { years }\end{array}$ & $\begin{array}{l}1.344 ; \text { GAD: not } \\
\text { reported }\end{array}$ & $28.13 \pm 4.26$ years & $\begin{array}{l}1.286 ; \text { GAD: not } \\
\text { reported }\end{array}$ & $\begin{array}{l}\text { delivery: PE group: } \\
12.3 \pm 3.17 \text { (Age: } \\
38.26 \pm 12.63 \text { ); Control } \\
\text { group: } 12.7 \pm 3.33 \\
\text { years (Age: } 39.54 \pm \\
10.26 \text { ) }\end{array}$ & $\begin{array}{l}\text { prepregnancy BMI, } \\
\text { smoking habits and } \\
\text { family history of } \\
\text { DM, CVD and } \\
\text { preeclampsia }\end{array}$ \\
\hline $\begin{array}{l}\text { Östlund E, } 2013 \\
\text { [53] }\end{array}$ & $\begin{array}{l}\text { Sweden; Prospective } \\
\text { cohort study }\end{array}$ & $\begin{array}{l}\text { No smoking and none used } \\
\text { oral contraceptives }\end{array}$ & $\begin{array}{l}\text { PE: } 15 \text { women with severe } \\
\text { preeclampsia; Age } 11 \text { years } \\
\text { after delivery: } 39.4 \pm 3.6 \text { years }\end{array}$ & $\begin{array}{l}\text { Parity: } 1.8 \pm 0.9 \\
\text { GAD: } 245 \pm 6 \text { days }\end{array}$ & $\begin{array}{l}\text { Control: } 16 \text { non-complicated } \\
\text { pregnant women; Age: } 11 \\
\text { years after delivery } 41.2 \pm \\
3.2 \text { years }\end{array}$ & $\begin{array}{l}\text { Parity: } 2.5 \pm 0.7 \\
\text { GAD: } 281 \pm 6 \text { days }\end{array}$ & $\begin{array}{l}\text { Study performed } 11.2 \\
\pm 0.6 \text { years following } \\
\text { the index pregnancy }\end{array}$ & $\begin{array}{l}\text { Age, parity and date } \\
\text { of delivery }\end{array}$ \\
\hline $\begin{array}{l}\text { Portelinha A, } 2008 \\
\text { [54] }\end{array}$ & $\begin{array}{l}\text { Portugal; } \\
\text { Retrospective cohort } \\
\text { study }\end{array}$ & $\begin{array}{l}\text { Prior history of hypertension, } \\
\text { heart disease, DM, renal } \\
\text { disease, infections, recent } \\
\text { surgery and current } \\
\text { pregnancy. None women were } \\
\text { postmenopausal. Patients with } \\
\text { hepatitis A or B were identified }\end{array}$ & $\begin{array}{l}\text { PE: } 58 \text { women according to } \\
\text { the ISSHP; Age: } 27[24,3]\end{array}$ & $\begin{array}{l}\text { Parity: not reported. } \\
\text { GAD: } 34[33,37] \\
\text { weeks; Cesarean } \\
\text { rate: } 74.1 \%\end{array}$ & $\begin{array}{l}48 \text { women without medical } \\
\text { complications associated to } \\
\text { pregnancy; Age: } 28[25,33]\end{array}$ & $\begin{array}{l}\text { Parity: not reported. } \\
\text { GAD: } 39[38,40] ; \\
\text { Cesarean rate: } 28.6 \%\end{array}$ & $\begin{array}{l}\text { Data at recall: women } \\
\text { with PE: Age } 34 \\
\text { [30, 39]; control group: } \\
34[31,39] \text { years. Years } \\
\text { since delivery: PE: } 6 \\
{[4,8] \text { years; Control }} \\
\text { group: } 6[4,8]\end{array}$ & $\begin{array}{l}\text { Age, BMI, time since } \\
\text { pregnancy, smoking, } \\
\text { contraceptive intake, } \\
\text { alcohol consumption }\end{array}$ \\
\hline $\begin{array}{l}\text { Portelinha A, } 2010 \\
\text { [55] }\end{array}$ & $\begin{array}{l}\text { Portugal; } \\
\text { Retrospective cohort } \\
\text { study }\end{array}$ & $\begin{array}{l}\text { Prior history of } \\
\text { hypertension, CVD, DM, } \\
\text { renal disease, infections, } \\
\text { recent surgery and current } \\
\text { pregnancy. None women } \\
\text { were postmenopausal }\end{array}$ & $\begin{array}{l}\text { PE: } 90 \text { women according to } \\
\text { the ISSHP; Age: } 28[24,32]\end{array}$ & $\begin{array}{l}\text { Parity: not reported; } \\
\text { GAD: } 35 \text { [32.5, 37.0] }\end{array}$ & $\begin{array}{l}\text { Control group } 60 \text { women; } \\
\text { Age: } 28[25,33]\end{array}$ & $\begin{array}{l}\text { Parity: not reported; } \\
\text { GAD: } 39.0[38.1,40.0]\end{array}$ & $\begin{array}{l}\text { Data at recall: women } \\
\text { with PE: Age } 34 \text { [31, } \\
\text { 39] years; control } \\
\text { group } 34[31,40] \text { years }\end{array}$ & Age \\
\hline Pouta A; 2004 [56] & $\begin{array}{l}\text { Finland; Retrospective } \\
\text { cohort study }\end{array}$ & Not found/not reported & $\begin{array}{l}\text { PE: } 49 \text { pregnant women } \\
\text { according to the ISSHP; Age: } \\
\text { Average } 25 \text { years }\end{array}$ & $\begin{array}{l}\text { Parity: Primiparous; } \\
\text { GAD: not reported }\end{array}$ & $\begin{array}{l}1369 \text { control pregnant } \\
\text { women; Age: average } 25 \\
\text { years }\end{array}$ & $\begin{array}{l}\text { Parity: Primiparous; } \\
\text { GAD: not reported }\end{array}$ & $\begin{array}{l}\text { The median interval } \\
\text { from first delivery to } \\
\text { examination at } 31 \\
\text { years was } 6 \text { years in all } \\
\text { groups [IQR: } 5 \text { months } \\
\text { to } 11 \text { years]. }\end{array}$ & Not matched \\
\hline $\begin{array}{l}\text { Romundstad PR; } \\
2010[57]\end{array}$ & $\begin{array}{l}\text { Norway; Retrospective } \\
\text { cohort study }\end{array}$ & $\begin{array}{l}\text { Pregnant women with } \\
\text { missing information on } \\
\text { essential measurements }\end{array}$ & $\begin{array}{l}\text { PE: } 168 \text { women with } \\
\text { preeclampsia according to } \\
\text { the ACOG criteria; Age: not } \\
\text { reported }\end{array}$ & $\begin{array}{l}\text { Parity: not reported; } \\
\text { GAD: not reported }\end{array}$ & $\begin{array}{l}\text { Control group: } 2964 \\
\text { normotensive pregnant } \\
\text { women; Age: not reported }\end{array}$ & $\begin{array}{l}\text { Parity: not reported; } \\
\text { GAD: not reported }\end{array}$ & $\begin{array}{l}\text { Recall for study } 21 \\
\text { years after delivery. }\end{array}$ & Not matched \\
\hline Sattar N, 2003 [58] & $\begin{array}{l}\text { United Kingdom; } \\
\text { Retrospective cohort }\end{array}$ & $\begin{array}{l}\text { No subject had any clinical } \\
\text { disease at the time of sampling }\end{array}$ & $\begin{array}{l}\text { PE: } 40 \text { primigravid women } \\
\text { with preeclampsia according }\end{array}$ & $\begin{array}{l}\text { Parity: Primiparous } \\
\text { women; GAD: } 36\end{array}$ & $\begin{array}{l}\text { Control: } 40 \text { uncomplicated } \\
\text { pregnant women matched as a }\end{array}$ & $\begin{array}{l}\text { Parity: Primiparous } \\
\text { women; GAD: } 40\end{array}$ & $\begin{array}{l}\text { Gravids delivering } \\
\text { between } 1975 \text { and }\end{array}$ & $\begin{array}{l}\text { Time of index } \\
\text { pregnancy, smoking, }\end{array}$ \\
\hline
\end{tabular}


Women with a history of hypertension, diabetes

PE: 70 women with PE according to the ACOG

including development of criteria; Age: $30.5+5.7$

gestational diabetes in any

pregnancy), renal disease, or

CVD were excluded

Spaan JJ, 2010 [60] Maastricht, The Netherlands;

None of the participants were PE: according to the using cholesterol-lowering Retrospetive cohort medication NHBPEPWG, N = 22 women; (27-43) weeks study
None of the women had a history of gestational DM cohort study

\section{Myocardial infarction,}

congestive heart failure, stroke, dementia, any cancer, autoimmune disease and neurological conditions

Women aged $<45$ or $>55$ years
Zoet GA, 2018 [63] The Netherlands: Retrospective cohor study

\section{Age: 23 (20-28) years \\ (a): $23(20-28)$ years}

PE: 48 women according the ACOG criteria; Age at first pregnancy: $34 \pm 8$ years

PE: 40 women with PE according to the ICD 9, who delivered between 1976 and 1982; Age: mean age 24 years

\section{PE: 164 women}

withhypertension + proteinuria; Age: not reported

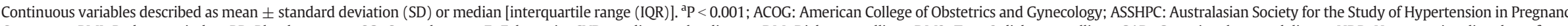

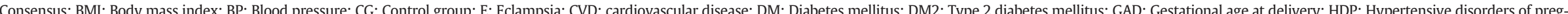

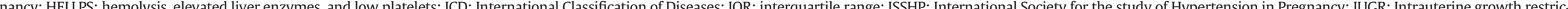
tion; NHBPEPWG: National High Blood Pressure Education Program Working Group; PE: Preeclampsia (Hypertension gestational + proteinuria) after 20 weeks of pregnancy; SEM: Standard error of the mean.

\section{Parity (\%):}

Age: $30.3 \pm 4.1$

pregnancy with PE 11

3.8.weeks

\section{Parity: parous}

Control: 29 women with

s; case
(27-43) weeks and controls $23(20-28)$ years
Parity (\%):

Primiparous 42

60.0; Previous

pregnancy with PE

1.6 weeks

Parity: parous 4/7 (38-42) weeks
1985. Ages at recall were in the PE group $43[40,47]$, and 44 $[43,47]$ years physical and biochemical

parameters at year 1 follow-up betwee women with and without preeclampsia Age at recall (23 years Matched for age, BMl after PE): PE group and date of delivery $49.0 \pm 3.9$ years group $49.8 \pm 3.9$ years (postmenopausal $\mathrm{n}=$ $5,17 \%)$

Parity: not reported; Control: 201 normotensive Parity: not reported; Age (years) at recall: Not matche pregnant women; Age at first GAD: not reported PE group $49 \pm 3.0$, pregnancy $28 \pm 1$ years

Parity: matched;

40 age- and parity-matched women without histories of preeclampsia, who delivered Parity: matched; $48.5 \pm 1.5$

Mean age of the study Parity and age at participants at the index birth; Mean index birth; Mean years

Parity: not reported; 387 women of similar age and Age and Parity: not Asymptomatic women, Age and ethnicity GAD: not reported ethnicity from the Multi-Ethnic reported; GAD: not Study of Atherosclerosis; Age: reported ged 45 to 55 years, 
Table 2

Meta-analyses of study outcomes.

\begin{tabular}{|c|c|c|c|c|}
\hline Outcomes & Number of studies (total sample) & Mean difference (MD) or risk difference (RD) and 95\%CI & P for effect & $\mathrm{I}^{2}$ \\
\hline Systolic blood pressure & $38(17,267)$ & $\mathrm{MD}=8.3 \mathrm{mmHg}(6.8$ to 9.7$)$ & $<0.00001$ & $78 \%$ \\
\hline Diastolic blood pressure & $37(17,232)$ & $\mathrm{MD}=6.8 \mathrm{mmHg}(5.6$ to 8.0$)$ & $<0.00001$ & $83 \%$ \\
\hline Hypertension & $12(2261)$ & $\mathrm{RD}=0.24 \%(0.15$ to 0.33$)$ & $<0.00001$ & $89 \%$ \\
\hline Body mass index & $34(17,039)$ & $\mathrm{MD}=2.0 \mathrm{~kg} / \mathrm{m}^{2}$ (1.6 to 2.4$)$ & $<0.00001$ & $56 \%$ \\
\hline Waist circumference & $13(11,371)$ & $\mathrm{MD}=4.3 \mathrm{~cm}(3.1$ to 5.5$)$ & $<0.00001$ & $31 \%$ \\
\hline Waist-to-hip ratio & $10(2364)$ & $\mathrm{MD}=0.02(0.01$ to 0.03$)$ & 0.004 & $59 \%$ \\
\hline Weight & $5(422)$ & $\mathrm{MD}=5.1 \mathrm{~kg}(2.2$ to 7.9$)$ & 0.0005 & $0 \%$ \\
\hline Lipoprotein (a) & $4(445)$ & $\mathrm{MD}=1.9 \mathrm{mg} / \mathrm{dL}(-0.8$ to 4.7$)$ & 0.17 & $7 \%$ \\
\hline Total cholesterol & $29(13,477)$ & $\mathrm{MD}=4.6 \mathrm{mg} / \mathrm{dL}(1.5$ to 7.7$)$ & 0.003 & $56 \%$ \\
\hline HDL-cholesterol & $29(13,367)$ & $\mathrm{MD}=-2.1 \mathrm{mg} / \mathrm{dL}(-3.5$ to -0.8$)$ & 0.001 & $57 \%$ \\
\hline LDL-cholesterol & $24(5220)$ & $\mathrm{MD}=4.6 \mathrm{mg} / \mathrm{dL}(0.2$ to 8.9$)$ & 0.04 & $81 \%$ \\
\hline VLDL-cholesterol & $3(162)$ & $\mathrm{MD}=0.3 \mathrm{mg} / \mathrm{dL}(-1.9$ to 2.5$)$ & 0.77 & $15 \%$ \\
\hline Triglycerides & $28(13,336)$ & $\mathrm{MD}=7.7 \mathrm{mg} / \mathrm{dL}$ (3.6 to 11.7 ) & 0.0002 & $46 \%$ \\
\hline Glucose & $25(4936)$ & $\mathrm{MD}=2.6 \mathrm{mg} / \mathrm{dL}(1.2$ to 4.0$)$ & 0.0003 & $78 \%$ \\
\hline Glycosylated hemoglobin & $10(9608)$ & $\mathrm{MD}=0.15 \%(-0.2$ to 0.5$)$ & 0.36 & $98 \%$ \\
\hline Insulin & $14(2337)$ & $\mathrm{MD}=19.1 \mathrm{pmol} / \mathrm{L}$ (11.9 to 26.2 ) & $<0.00001$ & $71 \%$ \\
\hline HOMA-IR & $14(1812)$ & $\mathrm{MD}=0.7(0.2$ to 1.2$)$ & 0.008 & $97 \%$ \\
\hline IGF-1 & $3(104)$ & $\mathrm{MD}=-15.1 \mathrm{ng} / \mathrm{mL}(-40.0$ to 9.8$)$ & 0.23 & $0 \%$ \\
\hline$C$ reactive protein & $11(1476)$ & $\mathrm{MD}=0.05 \mathrm{mg} / \mathrm{dL}(0.01$ to 0.09$)$ & 0.01 & $51 \%$ \\
\hline Microalbuminuria & $2(420)$ & $\mathrm{RD}=0.22 \%(-0.15$ to 0.59$)$ & 0.24 & $96 \%$ \\
\hline Albuminuria & $3(589)$ & $\mathrm{MD}=0.5 \mathrm{~g} / \mathrm{mol}$ creatinine $(-0.2$ to 1.2$)$ & 0.20 & $92 \%$ \\
\hline Metabolic syndrome & $5(265)$ & $\mathrm{RD}=0.11 \%(0.08$ to 0.15$)$ & $<0.000001$ & $0 \%$ \\
\hline
\end{tabular}

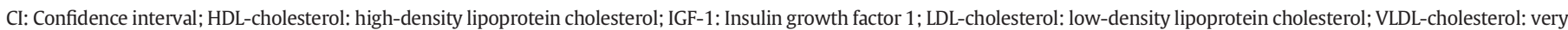
low-density lipoprotein cholesterol.

(ASSHPC) [18]. Definitions of PE severity, E and HELLP syndrome were equivalent for all these different scientific organizations: occurrence of hypertension (systolic blood pressure (SBP) $\geq 140 \mathrm{mmHg}$ and/or diastolic blood pressure (DBP) $\geq 90 \mathrm{mmHg}$ ) and proteinuria urinary albumin excretion $>300 \mathrm{mg} / 24 \mathrm{~h}$ or equivalent during the second half of pregnancy in gravid women

A

\begin{tabular}{|c|c|c|c|c|c|c|c|c|c|c|c|}
\hline Study or Subgroup & \multicolumn{3}{|c|}{ Pre-eclampsia } & \multicolumn{3}{|c|}{ Non pre-eclampsia } & Weight & $\begin{array}{l}\text { Mean Difference } \\
\text { IV, Random, } 95 \% \mathrm{CI}\end{array}$ & \multicolumn{3}{|c|}{$\begin{array}{c}\text { Mean Difference } \\
\text { IV, Random, } 95 \% \mathrm{CI}\end{array}$} \\
\hline Akhter 2013 & 120 & 7.41 & 48 & 110 & 7.41 & 58 & $3.7 \%$ & $10.00[7.17,12.83]$ & & & - \\
\hline Andersgaard 2012 & 138 & 12.25 & 901 & 130 & 8.65 & 7187 & $4.2 \%$ & $8.00[7.18,8.82]$ & & & - \\
\hline Aykas 2015 & 124.75 & 15.45 & 25 & 125.85 & 5.27 & 20 & $2.3 \%$ & $-1.10[-7.58,5.38]$ & & & \\
\hline Bar 1999 & 132.8 & 16.2 & 48 & 118 & 12 & 44 & $2.5 \%$ & $14.80[9.01,20.59]$ & & & \\
\hline Barden 1999 & 114 & 15.75 & 62 & 107 & 9.17 & 84 & $3.0 \%$ & $7.00[2.62,11.38]$ & & & \\
\hline Berends 2008 & 129 & 17.78 & 36 & 121.75 & 11.11 & 100 & $2.4 \%$ & $7.25[1.05,13.45]$ & & & \\
\hline Bokslag 2017 & 126 & 18.6 & 131 & 115 & 17 & 56 & $2.6 \%$ & $11.00[5.53,16.47]$ & & & \\
\hline Breetveld 2015 & 117 & 13 & 115 & 110 & 10 & 50 & $3.3 \%$ & $7.00[3.35,10.65]$ & & & \\
\hline Carleton 1988 & 123.7 & 19.9 & 23 & 114.4 & 19.9 & 23 & $1.1 \%$ & $9.30[-2.20,20.80]$ & & & \\
\hline Chambers 2001 & 116 & 16 & 78 & 103 & 10 & 48 & $3.0 \%$ & $13.00[8.46,17.54]$ & & & \\
\hline Christensen 2016 & 131 & 15 & 21 & 125 & 9 & 21 & $2.0 \%$ & $6.00[-1.48,13.48]$ & & & \\
\hline Coffeng 2010 & 135.3 & 13.4 & 17 & 114.7 & 6.3 & 16 & $2.1 \%$ & $20.60[13.52,27.68]$ & & & \\
\hline Dantas E.M 2013 & 116 & 7.1 & 10 & 108.5 & 7.9 & 17 & $2.5 \%$ & $7.50[1.71,13.29]$ & & & \\
\hline Drost 2012 & 127 & 16.91 & 339 & 119 & 13.01 & 332 & $3.9 \%$ & $8.00[5.72,10.28]$ & & & \\
\hline Freeman 2004 & 124.4 & 14.6 & 40 & 118.8 & 17.2 & 38 & $2.1 \%$ & $5.60[-1.50,12.70]$ & & & \\
\hline Garovic 2017 & 131.8 & 14.9 & 40 & 131.4 & 20.6 & 40 & $1.9 \%$ & $0.40[-7.48,8.28]$ & & & \\
\hline Girouard 2007 & 114.8 & 12.2 & 63 & 107.9 & 10.6 & 168 & $3.4 \%$ & $6.90[3.49,10.31]$ & & & \\
\hline Hamad 2007 & 111 & 10 & 18 & 103 & 8 & 17 & $2.4 \%$ & $8.00[2.02,13.98]$ & & & \\
\hline He 1999 & 120 & 16 & 25 & 108 & 8 & 24 & $2.1 \%$ & $12.00[4.96,19.04]$ & & & \\
\hline Hubel CA 2008 & 143 & 16 & 25 & 130 & 15 & 28 & $1.7 \%$ & $13.00[4.62,21.38]$ & & & \\
\hline Innes, K.E. 2005 & 113.8 & 13.34 & 13 & 107.1 & 13.34 & 13 & $1.3 \%$ & $6.70[-3.56,16.96]$ & & & \\
\hline Kvehaugen 2010 & 119.5 & 7.41 & 23 & 114.5 & 5.93 & 15 & $3.1 \%$ & $5.00[0.74,9.26]$ & & & \\
\hline Laivuori 1996 & 127 & 14.07 & 22 & 116 & 9.38 & 22 & $2.1 \%$ & $11.00[3.93,18.07]$ & & & \\
\hline Lampinen 2008 & 124.75 & 18.52 & 28 & 112 & 14.81 & 20 & $1.5 \%$ & $12.75[3.31,22.19]$ & & & \\
\hline Mangos 2012 & 121 & 20 & 39 & 115 & 12 & 35 & $2.0 \%$ & $6.00[-1.43,13.43]$ & & & \\
\hline Manten 2007 & 127 & 12 & 256 & 116 & 14 & 53 & $3.2 \%$ & $11.00[6.95,15.05]$ & & & \\
\hline McDonald 2013 & 119.5 & 16 & 109 & 115.8 & 14.4 & 219 & $3.4 \%$ & $3.70[0.14,7.26]$ & & & \\
\hline Nisell 1999 & 119 & 9.17 & 21 & 109 & 9.38 & 22 & $2.6 \%$ & $10.00[4.46,15.54]$ & & & \\
\hline Nohira 2013 & 127.6 & 18.8 & 58 & 122.2 & 14.2 & 61 & $2.4 \%$ & $5.40[-0.61,11.41]$ & & & \\
\hline Ostlund 2013 & 117 & 14 & 15 & 111 & 11 & 16 & $1.6 \%$ & $6.00[-2.90,14.90]$ & & & \\
\hline Portelinha 2010 & 121.5 & 13.33 & 90 & 119.25 & 12.59 & 60 & $3.1 \%$ & $2.25[-1.96,6.46]$ & & & \\
\hline Pouta 2004 & 123.5 & 14.81 & 49 & 117.5 & 10.37 & 1369 & $3.1 \%$ & $6.00[1.82,10.18]$ & & & \\
\hline Romundstad 2010 & 130 & 13.23 & 168 & 124 & 55.55 & 2964 & $3.7 \%$ & $6.00[3.17,8.83]$ & & & \\
\hline Sattar 2003 & 124.5 & 16.3 & 40 & 117.5 & 16.3 & 40 & $2.1 \%$ & $7.00[-0.14,14.14]$ & & & \\
\hline Smith 2009 & 120 & 11.9 & 70 & 111.3 & 9.3 & 70 & $3.4 \%$ & $8.70[5.16,12.24]$ & & & - \\
\hline Spaan 2010 & 132 & 17 & 22 & 117 & 15 & 29 & $1.6 \%$ & $15.00[6.04,23.96]$ & & & \\
\hline Suzuki, H. 2008 & 160 & 6 & 48 & 157 & 2 & 201 & $4.0 \%$ & $3.00[1.28,4.72]$ & & & $\leftarrow$ \\
\hline Zoet GA 2018 & 130 & 15 & 164 & 110 & 16 & 387 & $3.7 \%$ & $20.00[17.21,22.79]$ & & & \\
\hline Total $(95 \% \mathrm{CI})$ & & & 3300 & & & 13967 & $100.0 \%$ & $8.28[6.85,9.71]$ & & & \\
\hline $\begin{array}{l}\text { Heterogeneity. } \mathrm{Tau}^{2} \\
\text { Test for overall effect }\end{array}$ & 2.37, & $\mathrm{i}^{2}=$ & $59.29,0$ & 7 & 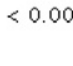 & $01 ; ; 1^{2}$ & 700 & & - & $\begin{array}{l}-10 \\
\text { Favors } \mathrm{PE}\end{array}$ & Favors 10 \\
\hline
\end{tabular}

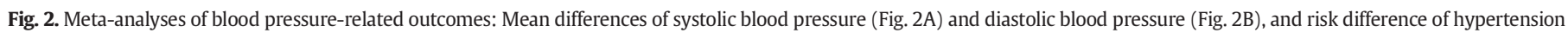
(Fig. 2C). A. Mean systolic blood pressure, $n=38$ studies $\left(I^{2}=78 \%\right.$ ). B. Mean diastolic blood pressure, $n=37$ studies $\left(I^{2}=83 \%\right)$. C. Hypertension, $n=12$ studies ( $\left.I^{2}=89 \%\right)$. 
B

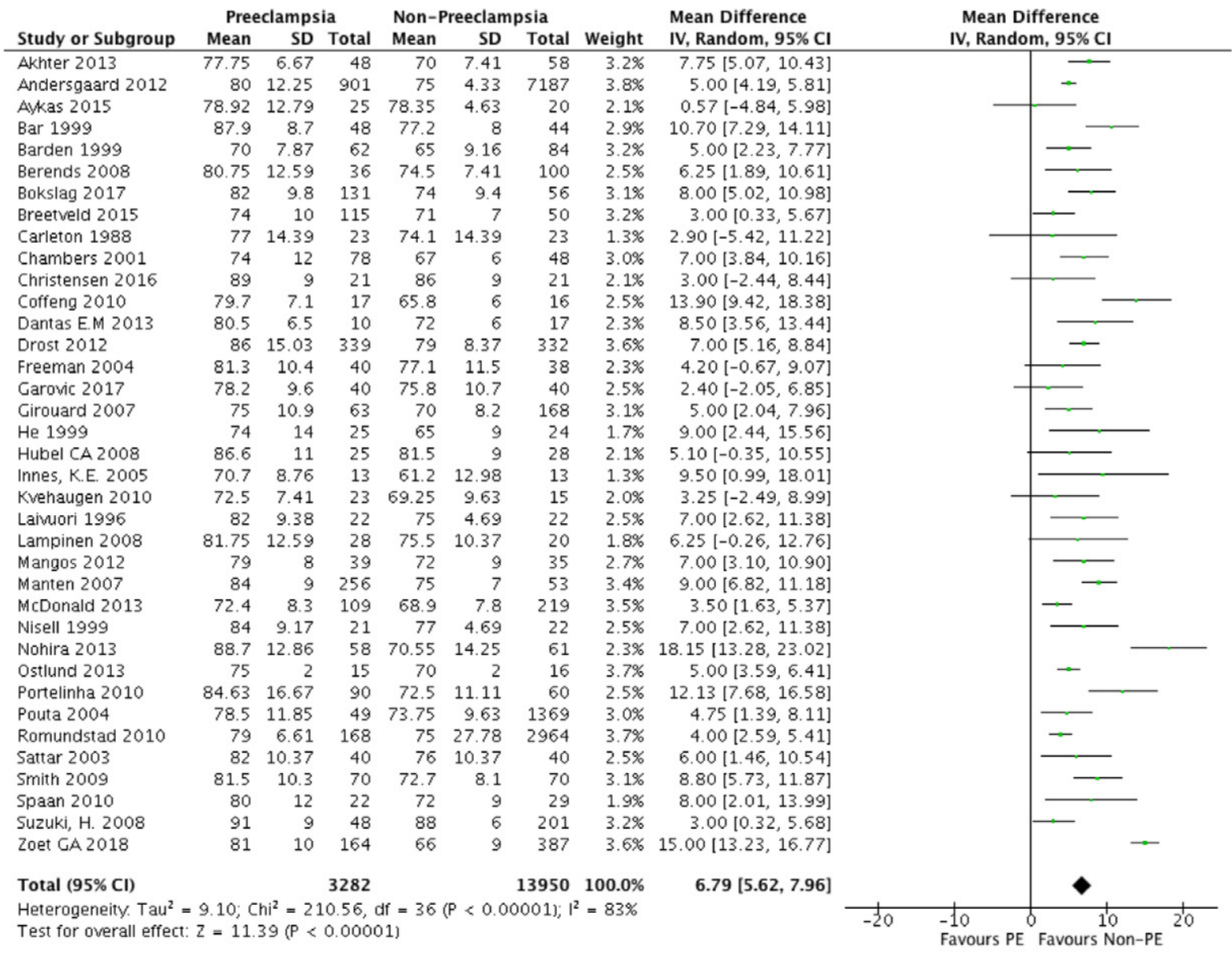

C

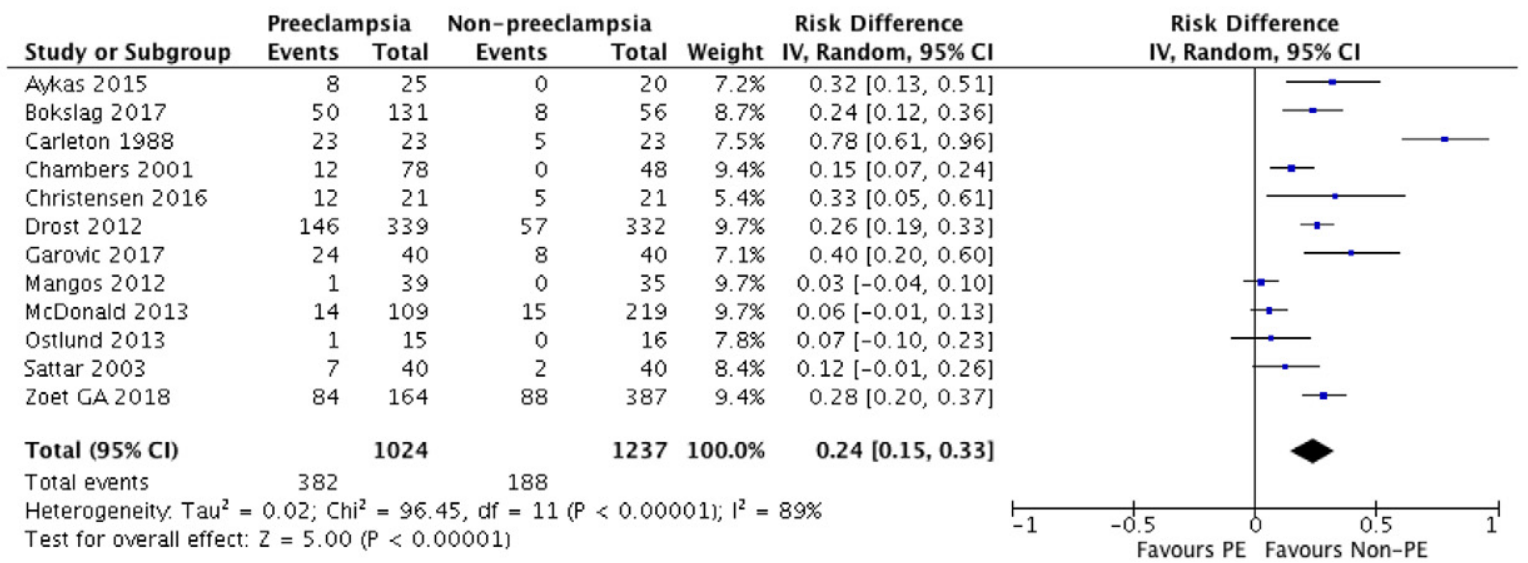

Fig. 2 (continued).

without previous hypertension or kidney disease. Proteinuria may be visually assessed by dipstick or by an automated device and confirmed by a $24 \mathrm{~h}$ urine collection.

\subsection{Outcomes of interest}

Outcomes of interest were metabolic and cardiovascular biomarkers measured at least three months after delivery, such as SBP, DBP, hypertension, body mass index (BMI), waist, waist-to-hip ratio (WHR), weight, lipoprotein (a) [Lp(a)], total cholesterol, high density lipoprotein cholesterol (HDL), low density lipoprotein cholesterol (LDL), very low density lipoprotein cholesterol (VLDL), triglycerides, glucose, glycosilated hemoglobin (HbA1c), insulin, homeostatic model assessment insulin resistance (HOMA-IR), insulin growth factor 1 (IGF-1), C-reactive protein (CRP), microalbuminuria, albuminuria, and metabolic syndrome (MetS). We extracted MetS definitions as provided by study authors, which may or not be based on published guidelines or consensus.

\subsection{Study selection and data extraction}

We included prospective or retrospective observational studies evaluating singleton PE and/or E and without previous kidney diseases. 
Normotensive uncomplicated pregnancies reported in the respective publication were considered as control groups. Published studies were eligible for inclusion if they reported metabolic or biochemical outcomes of interest three months after delivery or later. Exclusion criteria were: (a) PE data was not available or could not be extracted from the study groups; (b) no appropriate control group; (c) other hypertensive disorders different from PE, E and HELLP syndrome; (d) chronic pregestational diseases; and (e) metabolic or biochemical outcomes of interest measured within three months after delivery. Three of the authors (VAV, FRPL, YL) independently evaluated full-text articles for compliance with inclusion and exclusion criteria. Disagreements were managed through discussion with the other authors (FRPL, AVH) to reach a consensus. Authors were contacted if supplementary information or clarification was required in order to analyze study eligibility.

Extracted data included year of publication, country(ies) of study conduction, sample size for preeclampsia and control groups, time of follow up, baseline patient characteristics, outcomes per group, and variables used for confounder adjustment. Data extraction was also independently performed by 2 authors (VAV, YL) and disagreements were solved by discussion with all authors.

A

\begin{tabular}{|c|c|c|c|c|c|c|c|c|c|}
\hline Study or Subgroup & \multicolumn{3}{|c|}{ Preeclampsia } & \multicolumn{3}{|c|}{ Non-preeclampsia } & Weight & $\begin{array}{l}\text { Mean Difference } \\
\text { IV, Random, 95\% CI }\end{array}$ & $\begin{array}{c}\text { Mean Difference } \\
\text { IV, Random, } 95 \% \mathrm{CI}\end{array}$ \\
\hline Akhter 2013 & 27.25 & 6.67 & 48 & 23.5 & 4.44 & 58 & $2.2 \%$ & $3.75[1.54,5.96]$ & \\
\hline Andersgaard 2012 & 26 & 3.06 & 901 & 25 & 25.95 & 7187 & $5.5 \%$ & $1.00[0.37,1.63]$ & - \\
\hline Aykas 2015 & 30.68 & 4.38 & 25 & 28.55 & 2.38 & 20 & $2.5 \%$ & $2.13[0.12,4.14]$ & \\
\hline Barden 1999 & 26.3 & 6.3 & 62 & 22.3 & 4.58 & 84 & $2.8 \%$ & $4.00[2.15,5.85]$ & \\
\hline Berends 2008 & 27.85 & 6.81 & 45 & 24.53 & 4.22 & 101 & $2.3 \%$ & $3.32[1.17,5.47]$ & \\
\hline Bokslag 2017 & 25.7 & 4.59 & 131 & 23.9 & 4 & 56 & $3.9 \%$ & $1.80[0.49,3.11]$ & \\
\hline Breetveld 2015 & 25.6 & 6.2 & 115 & 23.3 & 3 & 50 & $3.6 \%$ & $2.30[0.89,3.71]$ & \\
\hline Chambers 2001 & 25.7 & 5.3 & 78 & 23.9 & 3.9 & 48 & $3.2 \%$ & $1.80[0.19,3.41]$ & \\
\hline Christensen 2016 & 28.46 & 5.5 & 21 & 26.62 & 5.7 & 21 & $1.2 \%$ & $1.84[-1.55,5.23]$ & \\
\hline Coffeng 2010 & 29.9 & 6.5 & 17 & 24.4 & 3.4 & 16 & $1.1 \%$ & $5.50[1.99,9.01]$ & \\
\hline Dantas E.M 2013 & 28.5 & 1.5 & 10 & 25 & 2.7 & 17 & $3.3 \%$ & $3.50[1.92,5.08]$ & \\
\hline Drost 2012 & 26.9 & 4.7 & 339 & 26.2 & 6.51 & 332 & $5.0 \%$ & $0.70[-0.16,1.56]$ & - \\
\hline Freeman 2004 & 27 & 5 & 40 & 26.3 & 4 & 38 & $2.5 \%$ & $0.70[-1.30,2.70]$ & \\
\hline Garovic 2017 & 29.8 & 5.78 & 40 & 26.43 & 6.59 & 40 & $1.7 \%$ & $3.37[0.65,6.09]$ & \\
\hline Girouard 2007 & 26.9 & 5.6 & 63 & 24.7 & 4.4 & 168 & $3.4 \%$ & $2.20[0.67,3.73]$ & \\
\hline Hamad 2007 & 25 & 5 & 18 & 22 & 3 & 17 & $1.7 \%$ & $3.00[0.29,5.71]$ & \\
\hline Hubel CA 2008 & 27.5 & 4.2 & 25 & 25.7 & 4.4 & 28 & $2.1 \%$ & $1.80[-0.52,4.12]$ & \\
\hline Innes, K.E. 2005 & 28.8 & 6.13 & 13 & 23.6 & 3.24 & 13 & $1.0 \%$ & $5.20[1.43,8.97]$ & \\
\hline Kvehaugen 2010 & 25.1 & 6.52 & 23 & 24.6 & 4.59 & 15 & $1.1 \%$ & $0.50[-3.03,4.03]$ & \\
\hline Laivuori 1996 & 23.3 & 3.75 & 22 & 21.9 & 1.88 & 22 & $2.9 \%$ & $1.40[-0.35,3.15]$ & \\
\hline Lampinen 2008 & 25 & 4 & 28 & 25 & 4 & 20 & $2.1 \%$ & $0.00[-2.30,2.30]$ & \\
\hline Mangos 2012 & 25.6 & 4.3 & 39 & 23.7 & 2.8 & 35 & $3.2 \%$ & $1.90[0.26,3.54]$ & \\
\hline Manten 2007 & 26 & 6 & 256 & 23 & 3 & 53 & $4.4 \%$ & $3.00[1.91,4.09]$ & \\
\hline McDonald 2013 & 28.2 & 5.7 & 109 & 27.2 & 5.8 & 219 & $3.8 \%$ & $1.00[-0.32,2.32]$ & \\
\hline Nisell 1999 & 25.5 & 5.96 & 21 & 22.8 & 3.28 & 22 & $1.5 \%$ & $2.70[-0.19,5.59]$ & \\
\hline Nohira 2013 & 28.93 & 3.78 & 58 & 25.43 & 3.06 & 61 & $4.0 \%$ & $3.50[2.26,4.74]$ & \\
\hline Ostlund 2013 & 25.8 & 6.1 & 15 & 23.3 & 3.1 & 16 & $1.2 \%$ & $2.50[-0.94,5.94]$ & \\
\hline Portelinha 2010 & 26.73 & 4.22 & 90 & 24.68 & 3.93 & 60 & $3.8 \%$ & $2.05[0.73,3.37]$ & \\
\hline Pouta 2004 & 24.55 & 4.44 & 49 & 23.25 & 3.41 & 1369 & $4.0 \%$ & $1.30[0.04,2.56]$ & \\
\hline Romundstad 2010 & 26.4 & 3.31 & 168 & 25.5 & 8.33 & 2964 & $5.6 \%$ & $0.90[0.32,1.48]$ & - \\
\hline Sattar 2003 & 26.75 & 5.19 & 40 & 25.75 & 3.7 & 40 & $2.6 \%$ & $1.00[-0.98,2.98]$ & \\
\hline Smith 2009 & 29 & 7 & 70 & 26 & 5 & 70 & $2.5 \%$ & $3.00[0.98,5.02]$ & \\
\hline Suzuki, H. 2008 & 27 & 4 & 48 & 24 & 3 & 201 & $4.1 \%$ & $3.00[1.79,4.21]$ & \\
\hline Zoet GA 2018 & 28.1 & 6.2 & 164 & 27.6 & 6.7 & 387 & $4.2 \%$ & $0.50[-0.66,1.66]$ & \\
\hline Total $(95 \% \mathrm{Cl})$ & & & 3191 & & & 13848 & $100.0 \%$ & $2.02[1.61,2.43]$ & \\
\hline $\begin{array}{l}\text { Heterogeneity. Tau }{ }^{2} \\
\text { Test for overall effec }\end{array}$ & 68 & $h i^{2}=$ & 5.28 & $d f=3$ & 0 & $0011 ; 1^{2}=$ & $=56 \%$ & & $\begin{array}{cccc}1 & 1 & 1 & 1 \\
-4 & -2 & 0 & 2 \\
\text { Favours } & \text { PE Favou }\end{array}$ \\
\hline
\end{tabular}

B

\begin{tabular}{|c|c|c|c|c|c|c|c|c|c|c|}
\hline Study or Subgroup & \multicolumn{3}{|c|}{ Preeclampsia } & \multicolumn{3}{|c|}{ Non-preeclampsia } & Weight & $\begin{array}{l}\text { Mean Difference } \\
\text { IV, Random, 95\% CI }\end{array}$ & \multicolumn{2}{|c|}{$\begin{array}{c}\text { Mean Difference } \\
\text { IV, Random, } 95 \% \mathrm{CI}\end{array}$} \\
\hline Andersgaard 2012 & 87 & 15.31 & 901 & 84 & 43.25 & 7187 & $20.9 \%$ & $3.00[1.59,4.41]$ & & $\rightarrow-$ \\
\hline Berends 2008 & 90.15 & 20 & 45 & 77.43 & 8.52 & 101 & $3.4 \%$ & $12.72[6.64,18.80]$ & & \\
\hline Bokslag 2017 & 80.75 & 11.11 & 131 & 76.95 & 8.74 & 56 & $10.4 \%$ & $3.80[0.82,6.78]$ & & $\longrightarrow$ \\
\hline Christensen 2016 & 91.4 & 14.7 & 21 & 85.3 & 13 & 21 & $1.9 \%$ & $6.10[-2.29,14.49]$ & & \\
\hline Dantas E.M 2013 & 91.5 & 9.4 & 10 & 85 & 6.8 & 17 & $2.9 \%$ & $6.50[-0.16,13.16]$ & & \\
\hline Drost 2012 & 86.5 & 12.21 & 339 & 83.2 & 12.09 & 332 & $17.4 \%$ & $3.30[1.46,5.14]$ & & $\rightarrow$ \\
\hline Garovic 2017 & 97.08 & 11.63 & 40 & 87.38 & 15.04 & 40 & $3.6 \%$ & $9.70[3.81,15.59]$ & & \\
\hline Girouard 2007 & 82.5 & 12.5 & 63 & 76.9 & 10.1 & 168 & $8.5 \%$ & $5.60[2.16,9.04]$ & & \\
\hline Hamad 2007 & 81 & 12 & 18 & 74 & 4 & 17 & $3.6 \%$ & $7.00[1.14,12.86]$ & & \\
\hline Kvehaugen 2010 & 81.5 & 12.59 & 23 & 79.75 & 8.15 & 15 & $3.0 \%$ & $1.75[-4.84,8.34]$ & & \\
\hline McDonald 2013 & 88.6 & 12.7 & 109 & 84.7 & 11.6 & 219 & $11.1 \%$ & $3.90[1.06,6.74]$ & & $\longrightarrow$ \\
\hline Pouta 2004 & 80.33 & 12.81 & 49 & 76.88 & 9.26 & 1369 & $8.0 \%$ & $3.45[-0.17,7.07]$ & & \\
\hline Sattar 2003 & 82.55 & 11.29 & 40 & 79.82 & 9.97 & 40 & $5.4 \%$ & $2.73[-1.94,7.40]$ & & \\
\hline Total $(95 \% \mathrm{Cl})$ & & & 1789 & & & 9582 & $100.0 \%$ & $4.32[3.13,5.52]$ & & \\
\hline $\begin{array}{l}\text { Heterogeneity. Tau² } \\
\text { Test for overall effect }\end{array}$ & 1.26 & $h i^{2}=1$ & 7.35 , df & $=12$ & $1=01$ & $1^{2}=3$ & & & $\begin{array}{l}-10 \\
\text { Favou }\end{array}$ & Favours \\
\hline
\end{tabular}

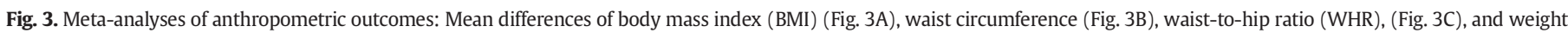
(Fig. 3d). A. BMI, $n=34$ studies $\left(I^{2}=56 \%\right)$. B. Waist circumference, $n=13$ studies $\left(I^{2}=31 \%\right)$. C. Waist-to-hip ratio, $n=10$ studies $\left(I^{2}=59 \%\right)$. D. Weight, $n=5$ studies $\left(I^{2}=0 \%\right)$ 
C

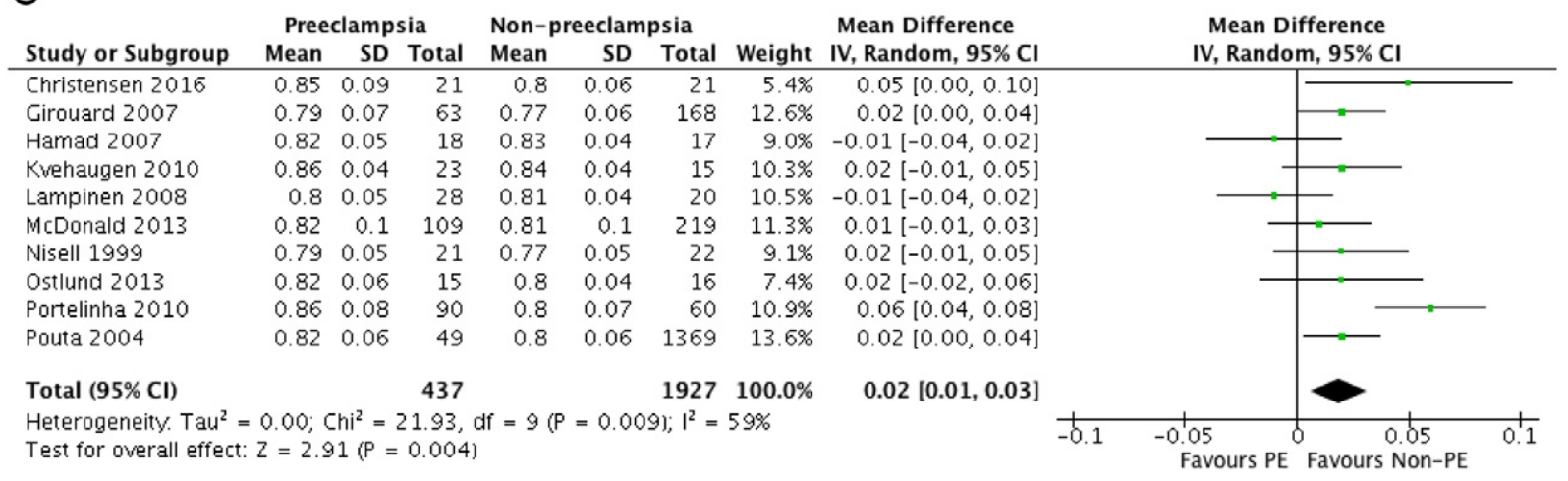

D

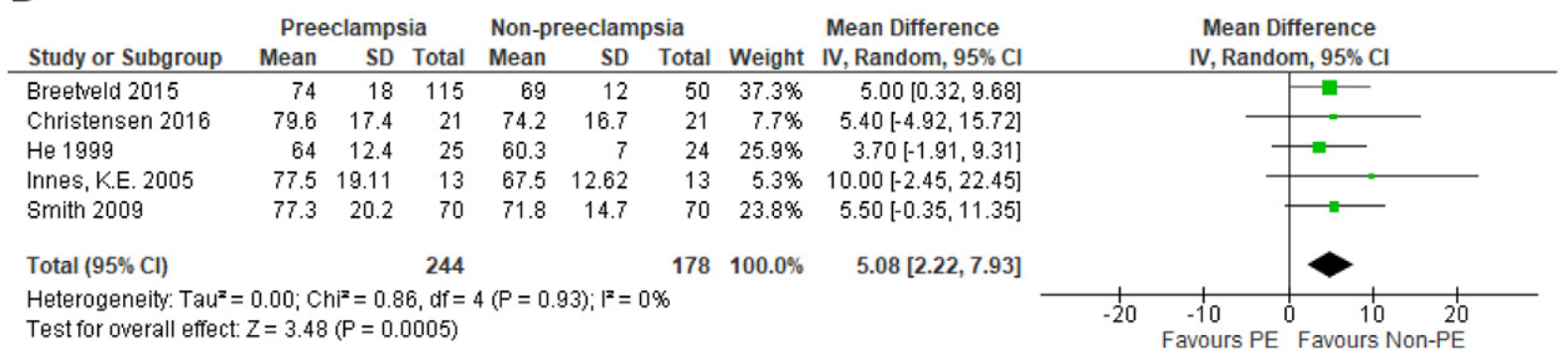

Fig. 3 (continued).

\subsection{Risk of bias assessment}

The risk of bias of selected studies was assessed independently by two authors (VP. and AVH) using the Newcastle-Ottawa scale (NOS) for cohort studies [19]. The NOS consists of three parameters of quality: selection, comparability and outcome assessment. The NOS assigns a maximum of four points for selection, two points for comparability and three points for exposure or outcome. NOS scores of $\geq 7$ were considered as high-quality studies and NOS scores of 5-6 were considered moderate quality. Any discrepancies were addressed by a reevaluation of the original article to reach consensus.

\subsection{Statistical analyses}

Inverse variance random-effects models were used for metaanalyses. Effects were reported as risk difference (RD) for dichotomous outcomes or mean differences (MD) for continuous outcomes and their 95\% CIs. Continuous outcomes were adjusted for baseline values per exposure arm. For studies reporting medians $(\mathrm{m})$ and interquartile ranges $(\mathrm{IQR})$, means were estimated by $\mathrm{x}=(\mathrm{a}+2 \mathrm{~m}+\mathrm{b}) / 4$, where $\mathrm{m}$ is median and a and b are P25 and P75, respectively [20]. SDs were estimated using $\mathrm{SD}=\mathrm{IQR} / 1.35$. When median and ranges were provided, the mean was estimated by $\mathrm{x}=(\mathrm{a}+2 \mathrm{~m}+\mathrm{b}) / 4$ using the values of the median $(\mathrm{m})$, the smallest and largest value ( $\mathrm{a}$ and $\mathrm{b}$, respectively); SD was estimated by $\mathrm{SD}=$ range $/ 4$ if sample size was $<70$ and $\mathrm{SD}=$ range $/ 6$ if sample size was $>70[20]$.

A p $<0.1$ for the Chi-square test was defined as an indicator of heterogeneity; a $\mathrm{Tau}^{2}>1$ was defined as the presence of substantial statistical heterogeneity. An $\mathrm{I}^{2}$ value of $0-30 \%$ was used to define low heterogeneity, $30-60 \%$ moderate heterogeneity, and $>60 \%$ substantial heterogeneity [21]. Potential publication bias was estimated by the Begg's funnel plot and the Egger's linear regression test [22].

We predefined subgroup analyses by (i) time of follow up, (ii) year of publication, and (iii) presence of adjustment for confounders. Statistical analyses were conducted using Review Manager (RevMan 5.3;
Cochrane Collaboration, Oxford, UK) and the Comprehensive Metaanalysis (Version 2; Biostat, Englewood, NJ).

\section{Results}

\subsection{Selection of studies}

A total of 2671 abstracts were identified through search engine and 14 additional full-papers were identified by manual search. After removal of duplicates, 2022 items were evaluated, of which 1966 did not fulfill inclusion criteria. Hence, 56 full texts were assessed for eligibility. Nine papers did not report separated information of $\mathrm{PE} / \mathrm{E}$ patients, five reported duplicate information, and one was a crosssectional study (Fig. 1). Finally, a total of 41 full papers [23-63] were evaluated for qualitative and quantitative assessment.

\subsection{Characteristics of included studies}

The 41 cohort studies included 3300 women who previously suffered PE/E and 13,967 controls. Six publications reported complementary information from three pairs of studies (first pair [37,40], second pair [39,62], and third pair [54,55]) (Table 1). PE/E sample sizes across studies ranged from 10 [35] to 901 [24]. Included studies described 207 women with HELLP syndrome in three studies, as compared with normotensive pregnant women $[29,35,49]$. Women were followed up from three months post-delivery $[32,49]$ to $>20$ years after delivery $[24,38,39,43,50,57,60,62]$. Publications included women from Europe [23-25,28-30,32-34,36,38,41-43,45-47,49,51,53-58,60,63], North America [31,37,39,40,44,50,59,62], and from other world regions $[26,27,35,48,52,61]$. There were no differences in baseline demographics and clinical characteristics between arms within each study (Table 1).

Diagnoses of PE and E were based on definitions from different scientific organizations such as ACOG ( $\mathrm{n}=14$ studies), ISSHP ( $\mathrm{n}=9$ studies), NHBPEWG ( $\mathrm{n}=4$ studies), ICD ( $\mathrm{n}=2$ studies), and ASSHPC ( $\mathrm{n}=1$ study), and standard clinical diagnosis of PE/E ( $\mathrm{n}=10$ studies) (Table 1). Other publications defined PE without referring to scientific 
societies and as an association of conventional hypertension and proteinuria developing after 20 weeks of pregnancy ( $\mathrm{n}=10$ studies) which remitted within a few days after delivery. All included studies had similar PE/E definitions: new onset hypertension and proteinuria after 20 weeks of gestation and in the case of $\mathrm{E}$ also included coma and/or seizures in previously normotensive women without renal pathology.

\subsection{Risk of bias assessment}

Using the NOS scale, all but one study [25] were identified as high quality (Appendix A, eSupplementary Table 1 ). All studies clearly identified the study population; patients were representative of average $\mathrm{PE} / \mathrm{E}$ cases and controls were derived from the same population as cases. In all studies, secure patient records were used for ascertainment of PE/E and assessment of outcomes. All studies had adequate follow-up time. Overall 23 studies identified important confounders or prognostic factors and were used for adjustment of the association between PE/E and cardiovascular risk. There was considerable variation in the selection of confounding variables for adjustment (Appendix A, eSupplementary Table 1). The most common confounder that was adjusted for was age.

\subsection{Meta-analyses of outcomes}

\subsubsection{Blood pressure}

In 38 studies ( $\mathrm{n}=17,267)$, SBP was significantly higher in women with previous diagnoses of $\mathrm{PE} / \mathrm{E}$ as compared to normotensive women (Table 2, Fig. 2A). In 37 studies ( $\mathrm{n}=17,232)$ DBP was significantly higher in women with previous diagnosis of PE/E (Table 2; Fig. 2B). In 12 studies ( $\mathrm{n}=2263)$, hypertension risk was significantly higher in women with PE/E (Table 2; Fig. 2C). There was high heterogeneity of effects on SBP and DBP across studies.

\subsubsection{Anthropometric outcomes}

In 34 studies ( $\mathrm{n}=17,039$ ) BMI was significantly higher in women with previous diagnosis of $\mathrm{PE} / \mathrm{E}$ (Table 2, Fig. 3A). In 13 studies ( $\mathrm{n}=11,371$ ) waist circumference was significantly higher in women with previous diagnosis of PE/E (Table 2, Fig. 3B). In 10 studies ( $\mathrm{n}=2364)$ the WHR was significantly higher in women with previous diagnosis of $\mathrm{PE} / \mathrm{E}$ (Table 2, Fig. 3C). In five studies $(n=422)$ weight was significantly higher in women with previous diagnosis of PE/E (Table 2; Fig. 3D). There was low to

A

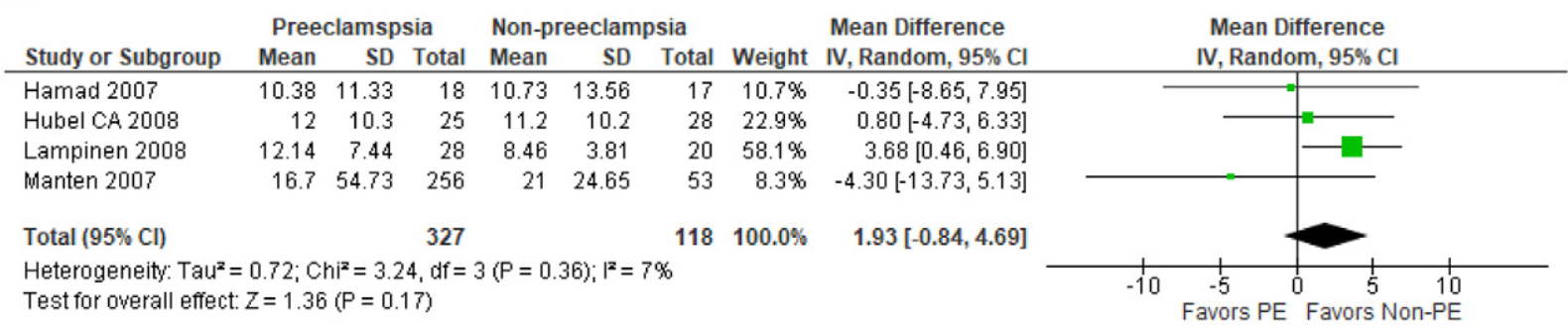

B

\begin{tabular}{|c|c|c|c|c|c|c|c|c|c|c|}
\hline \multirow[b]{2}{*}{ Study or Subgroup } & \multicolumn{3}{|c|}{ Preeclampsia } & \multicolumn{3}{|c|}{ Non-preelampsia } & \multirow{3}{*}{$\begin{array}{r}\text { Weight } \\
7.9 \%\end{array}$} & \multirow{3}{*}{$\begin{array}{l}\begin{array}{l}\text { Mean Difference } \\
\text { IV, Random, 95\% CI }\end{array} \\
3.09[-0.21,6.39]\end{array}$} & \multirow{2}{*}{\multicolumn{2}{|c|}{$\begin{array}{c}\text { Mean Difference } \\
\text { IV, Random, } 95 \% \mathrm{CI}\end{array}$}} \\
\hline & Mean & SD & Total & Mean & SD & Total & & & & \\
\hline Andersgaard 2012 & 236.66 & 47.32 & 901 & 233.57 & 50.17 & 7187 & & & & $E$ \\
\hline Barden 1999 & 211.91 & 79.13 & 62 & 184.46 & 52.98 & 84 & $1.5 \%$ & $27.45[4.73,50.17]$ & & \\
\hline Berends 2008 & 191.42 & 40.1 & 43 & 208.82 & 45.83 & 100 & $2.8 \%$ & $-17.40[-32.38,-2.42]$ & & \\
\hline Bokslag 2017 & 189.48 & 40.1 & 131 & 200.12 & 37.24 & 56 & $3.7 \%$ & $-10.64[-22.57,1.29]$ & & \\
\hline Breetveld 2015 & 180.1 & 28.6 & 115 & 187 & 29.7 & 50 & $4.6 \%$ & $-6.90[-16.65,2.85]$ & & \\
\hline Chambers 2001 & 186 & 35 & 78 & 182 & 35 & 48 & $3.5 \%$ & $4.00[-8.58,16.58]$ & & \\
\hline Christensen 2016 & 191.03 & 39.06 & 21 & 182.52 & 25.52 & 21 & $1.9 \%$ & $8.51[-11.45,28.47]$ & & \\
\hline Coffeng 2010 & 177.88 & 30.94 & 17 & 170.15 & 27.07 & 16 & $1.9 \%$ & $7.73[-12.08,27.54]$ & & \\
\hline Dantas E.M 2013 & 180.5 & 11.6 & 10 & 173 & 28.8 & 17 & $2.7 \%$ & $7.50[-7.96,22.96]$ & & \\
\hline Drost 2012 & 187.94 & 29.12 & 339 & 187.55 & 32.35 & 332 & $7.2 \%$ & $0.39[-4.27,5.05]$ & & \\
\hline Garovic 2017 & 190.5 & 34.81 & 40 & 203.38 & 30 & 40 & $3.0 \%$ & $-12.88[-27.12,1.36]$ & & \\
\hline Girouard 2007 & 184.46 & 39.44 & 63 & 175.56 & 35.58 & 168 & $4.0 \%$ & $8.90[-2.23,20.03]$ & & \\
\hline Hamad 2007 & 156.23 & 40.22 & 18 & 160.48 & 21.66 & 17 & $1.7 \%$ & $-4.25[-25.49,16.99]$ & & \\
\hline He 1999 & 186.18 & 27.94 & 24 & 167.35 & 31.81 & 24 & $2.4 \%$ & $18.83[1.89,35.77]$ & & \\
\hline Hubel CA 2008 & 227 & 44 & 25 & 216 & 36 & 28 & $1.6 \%$ & $11.00[-10.80,32.80]$ & & \\
\hline Innes, K.E. 2005 & 202.9 & 31.01 & 13 & 194.3 & 26.68 & 13 & $1.6 \%$ & $8.60[-13.64,30.84]$ & & \\
\hline Laivuori 1996 & 202.63 & 25.52 & 22 & 192.58 & 30.94 & 22 & $2.4 \%$ & $10.05[-6.71,26.81]$ & & \\
\hline Lampinen 2008 & 181.94 & 24.06 & 28 & 184.94 & 37.53 & 20 & $2.0 \%$ & $-3.00[-21.71,15.71]$ & & \\
\hline Mangos 2012 & 193.35 & 30.94 & 39 & 189.48 & 27.07 & 35 & $3.3 \%$ & $3.87[-9.35,17.09]$ & & \\
\hline Manten 2007 & 201.08 & 34.8 & 256 & 181.75 & 30.94 & 53 & $4.8 \%$ & $19.33[9.97,28.69]$ & & \\
\hline McDonald 2013 & 129.55 & 34.37 & 109 & 125.68 & 34.37 & 219 & $5.5 \%$ & $3.87[-4.03,11.77]$ & & \\
\hline Nisell 1999 & 193.35 & 35.42 & 21 & 174.02 & 36.35 & 22 & $1.7 \%$ & $19.33[-2.12,40.78]$ & & \\
\hline Ostlund 2013 & 182.52 & 45.24 & 15 & 185.62 & 29.39 & 16 & $1.1 \%$ & $-3.10[-30.15,23.95]$ & & \\
\hline Portelinha 2010 & 207.2 & 39.3 & 90 & 206.6 & 34.3 & 60 & $3.8 \%$ & $0.60[-11.28,12.48]$ & & \\
\hline Pouta 2004 & 186 & 25.91 & 49 & 183.68 & 32.48 & 1369 & $5.7 \%$ & $2.32[-5.14,9.78]$ & & \\
\hline Sattar 2003 & 197.22 & 34.37 & 40 & 183.68 & 45.83 & 40 & $2.2 \%$ & $13.54[-4.21,31.29]$ & & \\
\hline Spaan 2010 & 229.7 & 36.74 & 22 & 216.17 & 39.44 & 29 & $1.7 \%$ & $13.53[-7.49,34.55]$ & & \\
\hline Suzuki, H. 2008 & 226 & 12 & 48 & 217 & 2 & 201 & $7.8 \%$ & $9.00[5.59,12.41]$ & & - \\
\hline Zoet GA 2018 & 208.82 & 38.67 & 164 & 197.22 & 38.67 & 387 & $5.9 \%$ & $11.60[4.54,18.66]$ & & \\
\hline Total $(95 \% \mathrm{Cl})$ & & & 2803 & & & 10674 & $100.0 \%$ & $4.59[1.53,7.66]$ & & \\
\hline $\begin{array}{l}\text { Heterogeneity. } \mathrm{Tau}^{2} \\
\text { Test for overall effect }\end{array}$ & $8.22 ; \mathrm{C}$ & $\begin{aligned} \mathrm{hi}^{2} & =6 \\
(\mathrm{P} & =0\end{aligned}$ & $\begin{array}{l}61, \mathrm{df} \\
0031\end{array}$ & $=28$ & $=0.0$ & $1) ; 1^{2}=$ & $56 \%$ & & $\begin{array}{c}-100 \\
\text { Favours PE }\end{array}$ & $\begin{array}{c}10 \\
\text { Favours }\end{array}$ \\
\hline
\end{tabular}

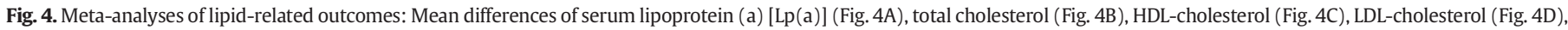

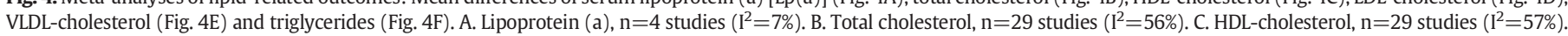
D. LDL-cholesterol, $n=24$ studies $\left(I^{2}=81 \%\right)$. E. VLDL-cholesterol, $n=3$ studies $\left(I^{2}=15 \%\right)$. F. Triglycerides, $n=28$ studies $\left(I^{2}=46 \%\right)$. 
C

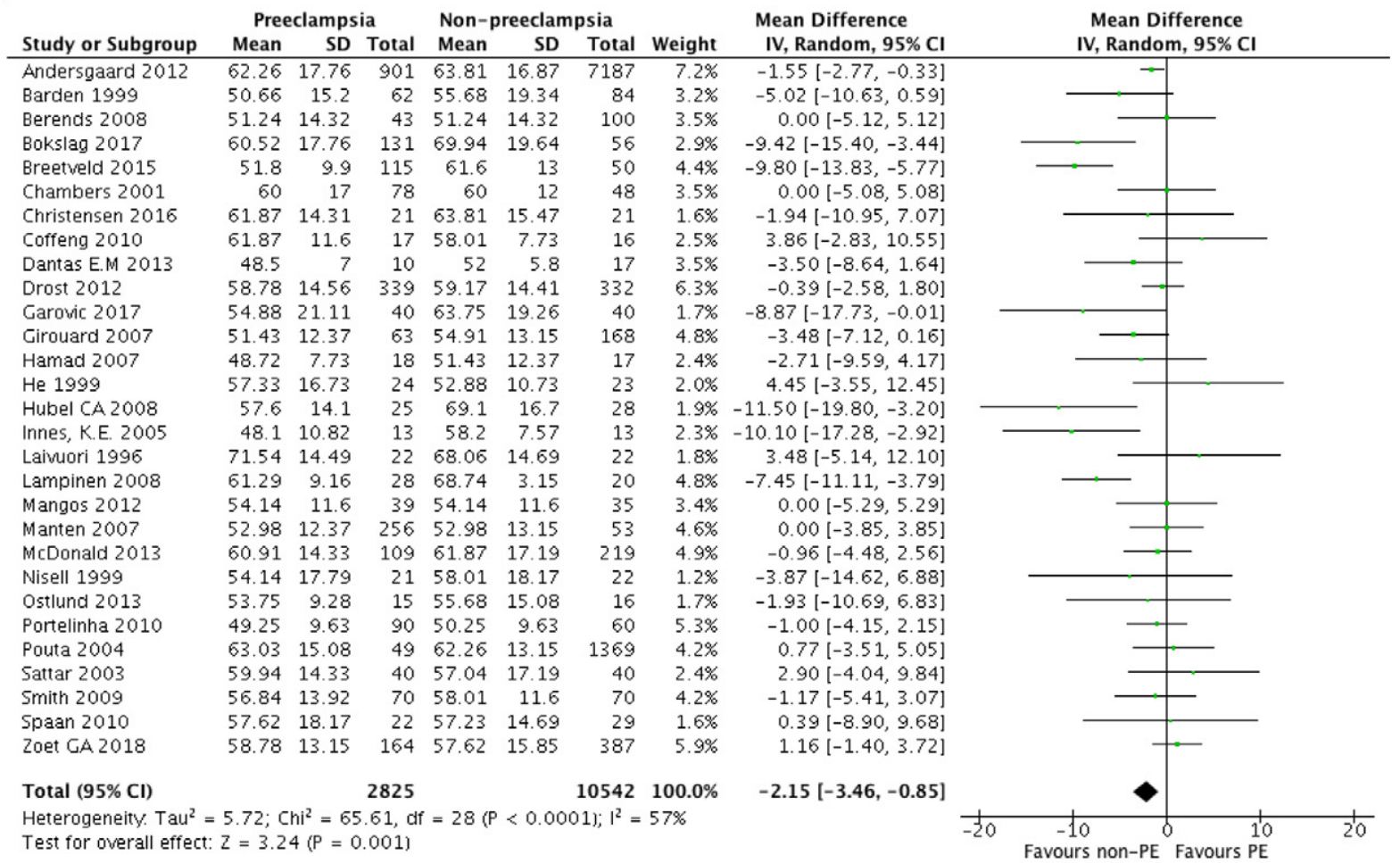

\section{D}

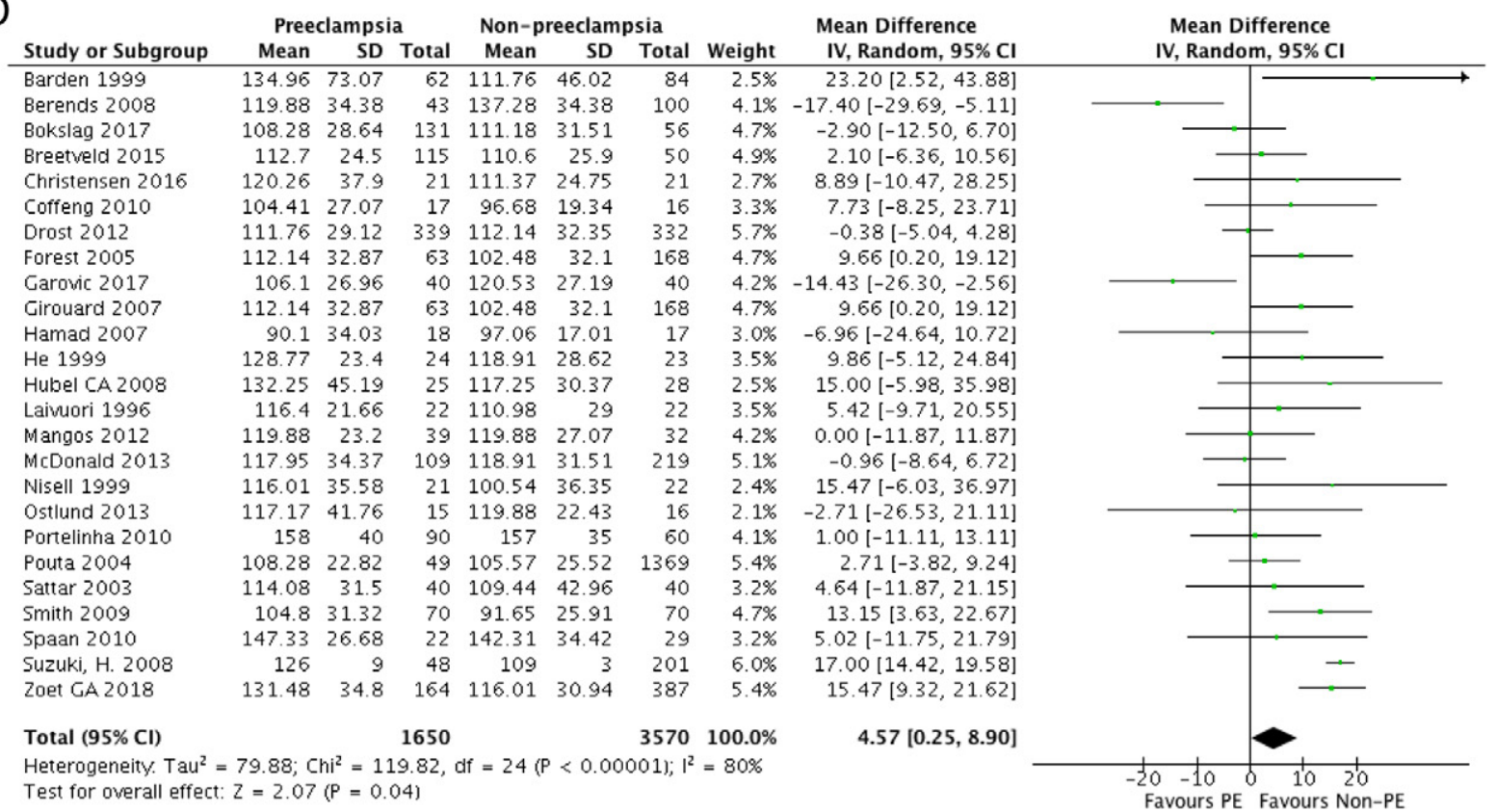

Fig. 4 (continued).

moderate heterogeneity of effects on anthropometric outcomes across studies.

\subsubsection{Lipid-related outcomes}

In 4 studies $(n=445)$ there was no significant difference in Lp (a) levels between women with and without PE/E (Table 2; Fig. 4A). In 29 studies ( $\mathrm{n}=13,477)$ TC was significantly higher in women with previous PE/E (Table 2; Fig. 4B). In 29 studies ( $\mathrm{n}=13,367)$ HDL was significantly lower in those with previous PE/E (Table 2; Fig. 4C). In 24 studies $(\mathrm{n}=5220)$ LDL was significantly higher in women with previous PE/E (Table 2; Fig. 4D). In three studies $(\mathrm{n}=162)$ there was no difference in VLDL in women with previous PE/E (Table 2; Fig. 4E). In 28 studies $(\mathrm{n}=13,336)$ triglycerides were higher in women with previous PE/E (Table 2; Fig. 4F). There was low to high heterogeneity of effects on lipid outcomes across studies.

\subsubsection{Glucose- and insulin-related outcomes}

In 25 studies $(n=4936)$ serum glucose was significantly higher in women with PE/E (Table 2, Fig. $5 A$ ). In 10 studies $(n=9608)$ there was no effect on HbA1c (Table 2; Fig. 5B). In 14 studies $(n=2327$ ) 
$E$

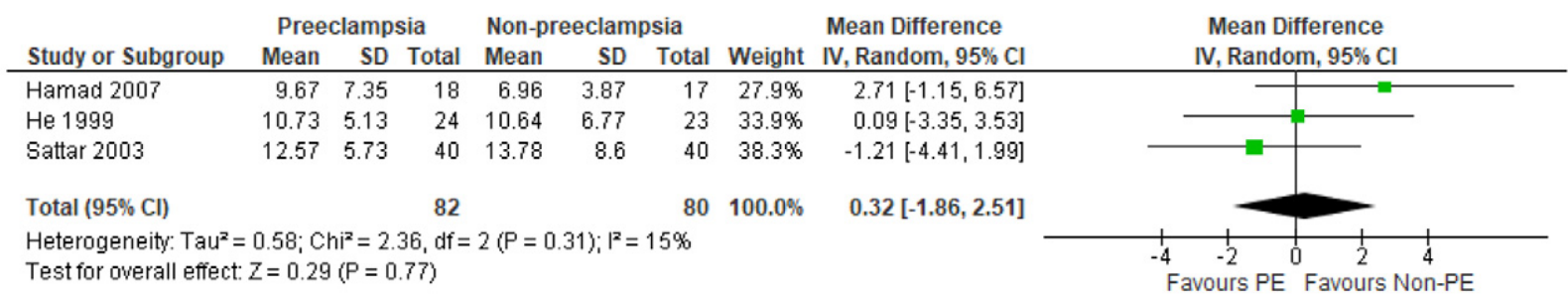

$\mathrm{F}$

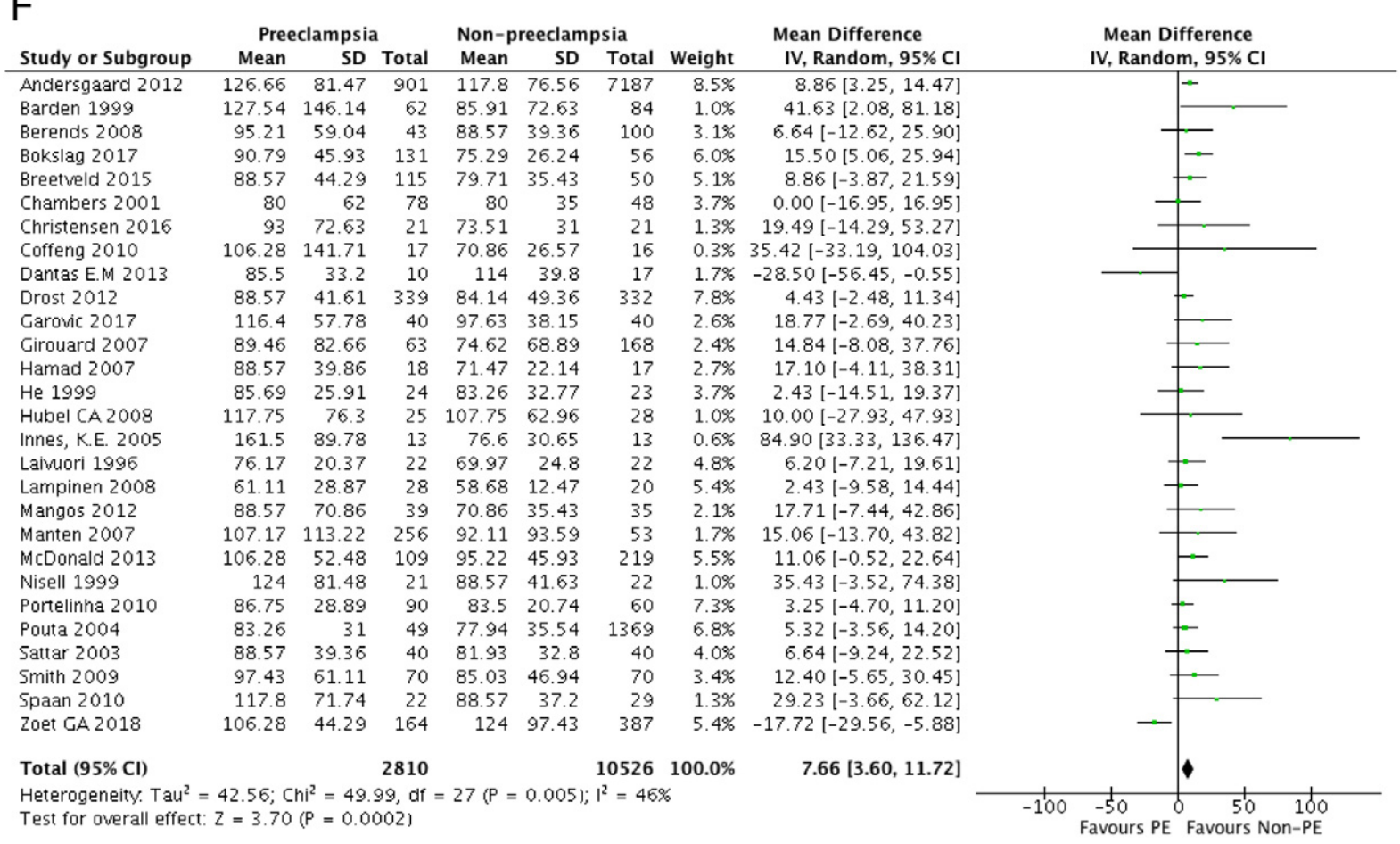

Fig. 4 (continued).

serum insulin was significantly higher in women with PE/E (Table 2; Fig. 5C). In 14 studies $(n=1812)$ the HOMA-IR index was significantly higher in women with PE/E (Table 2; Fig. 5D). In three studies $(n=104)$ there was no effect on IGF-1 in women with PE/E (Table 2; Fig. 5E). There was high heterogeneity of effects on glucose-related outcomes across studies.

\subsubsection{Other outcomes}

In 11 studies ( $\mathrm{n}=1376)$ CRP levels were significantly higher in women with PE/E (Table 2; Fig. 6a). In two studies $(n=428)$, there was no significant difference in microalbuminuria risk between women with and without PE/E (Table 2; Fig. 6b). In three studies $(n=589)$, there was no significant difference in albuminuria levels between women with and without PE/E (Table 2; Fig. 6c). In five studies ( $\mathrm{n}=$ 1688), the risk of MetS was significantly higher in women with PE/eclampsia (Table 2; Fig. 6d). There was low to high heterogeneity of effects across studies.

\subsection{Subgroup analyses}

Evaluation of subgroup effects by year of publication, time of follow up and adjustment of effects for confounders provided similar results as main analyses (Appendix A, eSupplementary eFigures 1 to 8 ).

\subsection{Publication bias}

Funnel plots of outcomes available in $>10$ studies showed that there was no asymmetry of points, except for triglycerides and glucose where small studies (i.e. those with larger SEs) were absent.

\section{Discussion}

\subsection{Main findings}

We found that women with PE/E or HELLP syndrome in comparison to women with normotensive pregnancies had later in life (i) higher hypertension risk and BP levels; (ii) higher BMI, waist circumference, waist-to-hip ratio, and weight, (iii) higher levels of total cholesterol, LDL, and triglycerides and lower levels of HDL; (iv) higher levels of serum glucose, insulin, the HOMA-IR index, $C$ reactive protein, and (v) higher risk of MetS. These results were based in cohort studies with low risk of bias (only one publication has high risk of bias), although heterogeneity of studies was high for several outcomes. In this study, we only included women with PE/E and/or HELLP syndrome as defined by medical and scientific organizations, since other hypertensive disorders of pregnancy may have a different pathophysiology, are heterogeneous in their clinical characteristics and managements, or 
have different specific therapies during early pregnancy or before pregnancy.

\subsection{What is known in the literature about the research question}

$\mathrm{PE} / \mathrm{E}$ is a heterogeneous multisystem disorder appearing during the second half of pregnancy in women without previous hypertension and proteinuria or renal disease, and is characterized by new onset hypertension and proteinuria after 20 weeks of pregnancy; PE/E is associated with high risks of preterm birth, intrauterine growth restriction, abruptio placentae, perinatal mortality and maternal morbidity and mortality. The degree of hypertension and proteinuria and the existence of other accompanying findings are variable [1,6,64-66]. PE/E risk factors include nulliparity, multifetal pregnancy, family history of PE, prior pregnancy complicated with placental insufficiency, excessive pre-pregnancy BMI, advanced maternal age, and use of assisted reproductive techniques $[1,6]$.

The Cardiovascular Risk in Young Finns Study linked data from primiparous women with pre-conceptional lipid metabolism, blood pressure and insulin and glucose metabolism, showing that high levels of triglycerides were associated with increased risk of PE and gestational diabetes [67]. Therefore, some pre-gestational metabolic alterations may in part contribute to the risk of $\mathrm{PE}$ and may persist after pregnancy. Also, women with PE have higher risk of developing later diabetes, hypertension and cardiovascular risk factors, especially when the hypertensive disorder occurred in late pregnancy or when there were two PE episodes (i.e. in two different pregnancies) [68]. Women with $\mathrm{PE} / \mathrm{E}$ have increased prevalence of subsequent hypertension, dyslipidemia, diabetes, congestive heart failure, stroke, renal and other subclinical alterations [8,12,13,69-71].

Our systematic review and meta-analyses identified hypertension, altered metabolic, and endocrine changes in women with PE/E as compared to women with normotensive pregnancies, before severe clinical complications are diagnosed. Risks of metabolic, anthropometric, glucose- and insulin-related outcomes, and hypertension and MetS reported in our study are intermediate risk factors of cardiovascular disease and type 2 diabetes mellitus. Lipid differences reported here suggest that women at risk of $\mathrm{PE} / \mathrm{E}$ have a trend for abnormal

A

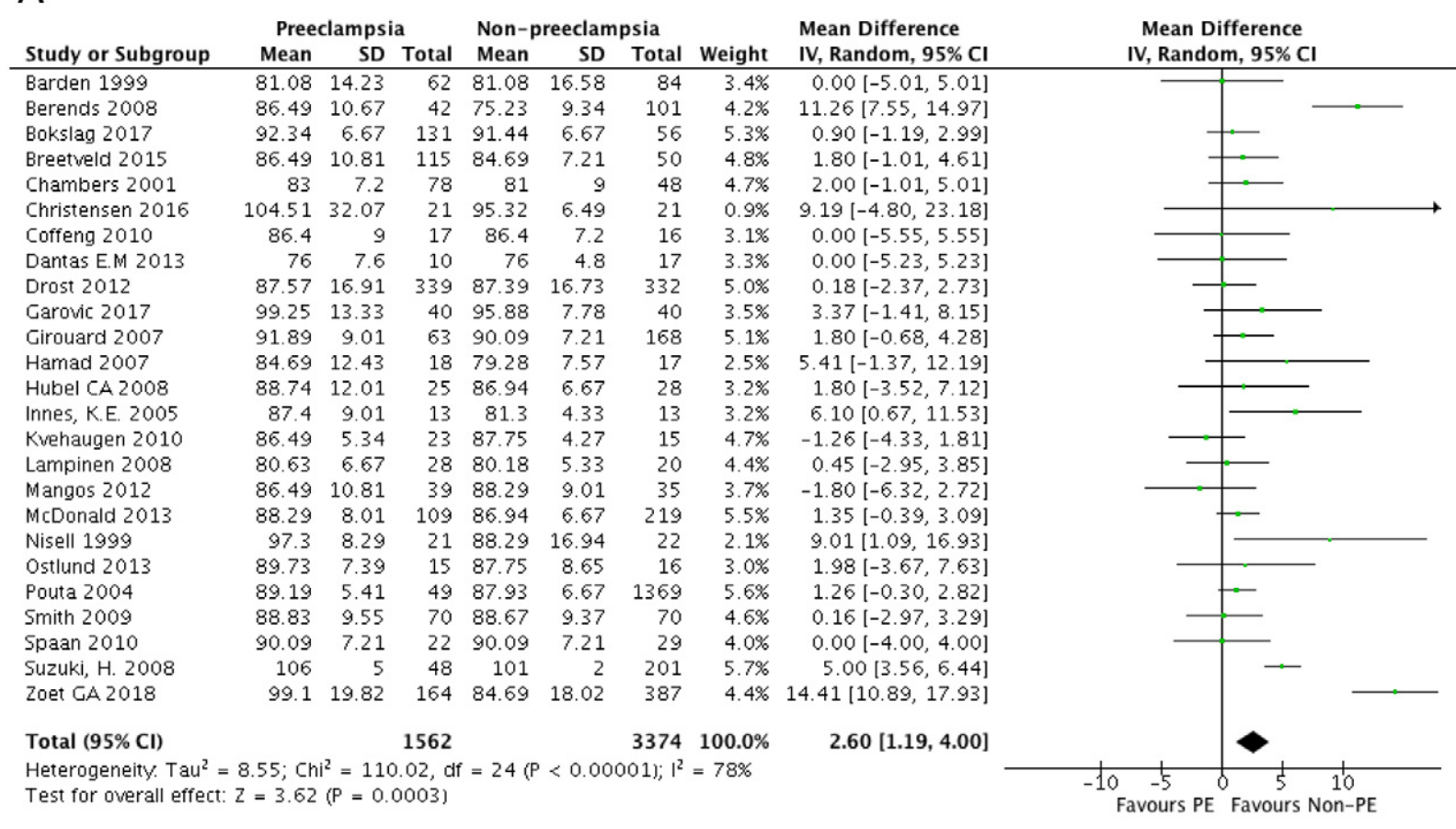

B

\begin{tabular}{|c|c|c|c|c|c|c|c|c|c|c|c|}
\hline \multirow[b]{2}{*}{ Study or Subgroup } & \multicolumn{3}{|c|}{ Preeclampsia } & \multicolumn{3}{|c|}{ Non-preeclampsia } & \multirow[b]{2}{*}{ Weight } & \multirow{2}{*}{$\begin{array}{l}\text { Mean Difference } \\
\text { IV, Random, } 95 \% \mathrm{Cl}\end{array}$} & \multirow{2}{*}{\multicolumn{2}{|c|}{$\begin{array}{c}\text { Mean Difference } \\
\text { IV, Random, } 95 \% \mathrm{Cl}\end{array}$}} & \\
\hline & Mean & SD & Total & Mean & SD & Total & & & & & \\
\hline Andersgaard 2012 & 5.48 & 1.68 & 901 & 5.43 & 1.3 & 7187 & $11.2 \%$ & $0.05[-0.06,0.16]$ & & $F$ & \\
\hline Bokslag 2017 & 5.4 & 0.3 & 131 & 5.23 & 0.22 & 56 & $11.3 \%$ & $0.17[0.09,0.25]$ & & $=$ & \\
\hline Christensen 2016 & 5.5 & 2.8 & 21 & 5.2 & 2.4 & 21 & $3.2 \%$ & $0.30[-1.28,1.88]$ & & & \\
\hline Coffeng 2010 & 4.68 & 1.03 & 17 & 5.2 & 0.15 & 16 & $9.1 \%$ & $-0.52[-1.02,-0.02]$ & & & \\
\hline Drost 2012 & 5.27 & 0.47 & 339 & 4.24 & 0.46 & 332 & $11.3 \%$ & $1.03[0.96,1.10]$ & & & $=$ \\
\hline Lampinen 2008 & 5.2 & 0.3 & 28 & 5.1 & 0.3 & 20 & $11.0 \%$ & $0.10[-0.07,0.27]$ & & & \\
\hline McDonald 2013 & 5.53 & 0.37 & 109 & 5.6 & 0.3 & 219 & $11.3 \%$ & $-0.07[-0.15,0.01]$ & 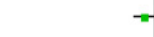 & & \\
\hline Sattar 2003 & 4.75 & 0.59 & 40 & 4.5 & 0.15 & 40 & $11.0 \%$ & $0.25[0.06,0.44]$ & & - & \\
\hline Spaan 2010 & 5.6 & 0.4 & 22 & 5.6 & 0.3 & 29 & $10.9 \%$ & $0.00[-0.20,0.20]$ & & - & \\
\hline White 2016 & 5.7 & 1.3 & 40 & 5.5 & 0.4 & 40 & $9.6 \%$ & $0.20[-0.22,0.62]$ & & & \\
\hline Total $(95 \% \mathrm{Cl})$ & & & 1648 & & & 7960 & $100.0 \%$ & $0.15[-0.18,0.49]$ & & & \\
\hline $\begin{array}{l}\text { Heterogeneity: } \mathrm{Tau}^{2} \\
\text { Test for overall effect }\end{array}$ & $\begin{array}{l}0.25 ; \mathrm{Cr} \\
\mathrm{Z}=0.91\end{array}$ & $\begin{array}{l}\mathrm{i}^{2}=54 \\
(\mathrm{P}=0\end{array}$ & $\begin{array}{l}45.30, \mathrm{~d} \\
.36)\end{array}$ & $f=9(P$ & 1.0000 & $; 1^{2}=0$ & $98 \%$ & & $\begin{array}{cc}1 & 1 \\
-1 & -0.5 \\
\text { Favours PE }\end{array}$ & $\begin{array}{cc}0 & 0.5 \\
\text { Favours n }\end{array}$ & 7 \\
\hline
\end{tabular}

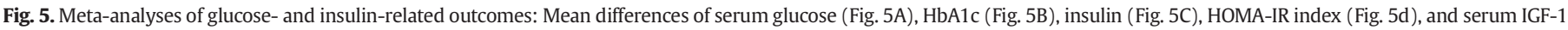

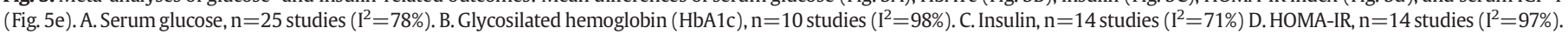
E. IGF- $1, n=3$ studies $\left(I^{2}=0 \%\right)$. 
C

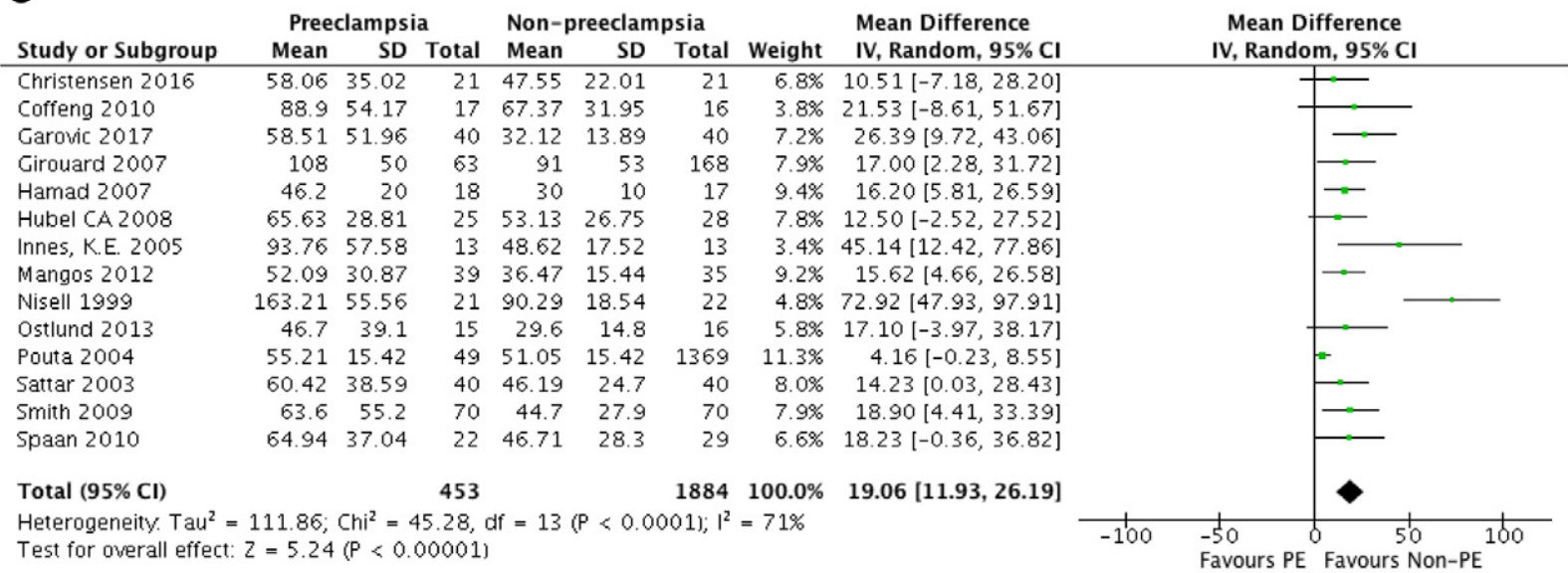

D

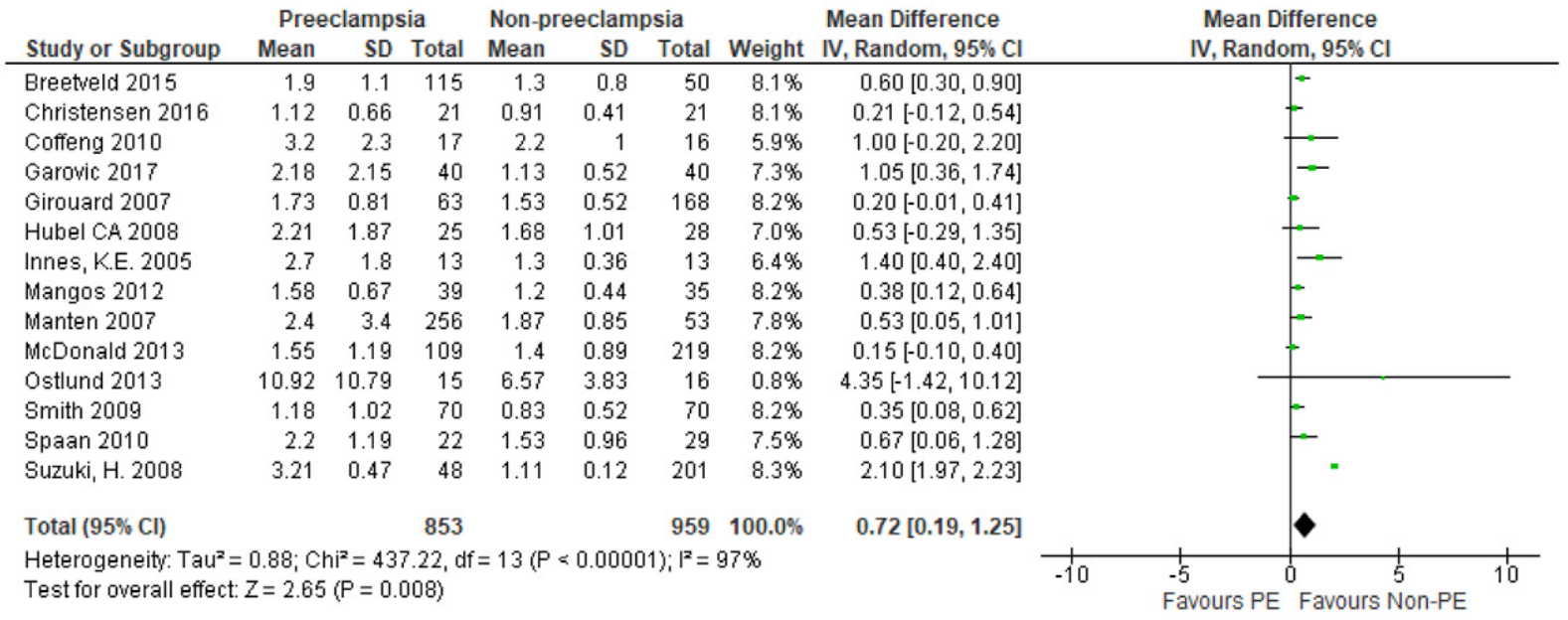

E

\begin{tabular}{|c|c|c|c|c|c|c|c|c|c|c|c|}
\hline \multirow[b]{2}{*}{ Study or Subgroup } & \multicolumn{3}{|c|}{ Preeclampsia } & \multicolumn{3}{|c|}{ Non-preeclampsia } & \multirow[b]{2}{*}{ Weight } & \multirow{2}{*}{$\begin{array}{l}\text { Mean Difference } \\
\text { IV, Random, } 95 \% \mathrm{Cl}\end{array}$} & \multirow{2}{*}{\multicolumn{2}{|c|}{$\begin{array}{l}\text { Mean Difference } \\
\text { IV, Random, } 95 \% \mathrm{Cl}\end{array}$}} & \\
\hline & Mean & SD & Total & Mean & $\mathrm{SD}$ & Total & & & & & \\
\hline Hamad 2007 & 221 & 54 & 18 & 240 & 72 & 17 & $34.6 \%$ & $-19.00[-61.35,23.35]$ & & \begin{tabular}{l|l}
-2 &
\end{tabular} & \\
\hline Innes, K.E. 2005 & 162.8 & 34.97 & 13 & 183.5 & 54.44 & 13 & $50.2 \%$ & $-20.70[-55.87,14.47]$ & & & \\
\hline Nisell 1999 & 342 & 109.98 & 21 & 330 & 103.19 & 22 & $15.2 \%$ & $12.00[-51.81,75.81]$ & & & \\
\hline Total $(95 \% \mathrm{Cl})$ & & & 52 & & & 52 & $100.0 \%$ & $-15.13[-40.04,9.78]$ & & & \\
\hline \multicolumn{9}{|c|}{$\begin{array}{l}\text { Heterogeneity: } \text { Tau }^{2}=0.00 ; \mathrm{Chi}^{2}=0.82, \mathrm{df}=2(\mathrm{P}=0.66) ; \mathrm{I}^{2}=0 \% \\
\text { Test for overall effect: } Z=1.19(\mathrm{P}=0.23)\end{array}$} & -100 & $\begin{array}{ccc}-50 & 0 & 50 \\
\text { avours PE } & \text { Favours }\end{array}$ & $\begin{array}{l}100 \\
n-P E\end{array}$ \\
\hline
\end{tabular}

Fig. 5 (continued).

adjustment during pregnancy. Recent evidence from a populationbased prospective cohort suggested that women with high blood pressure during pregnancy and postpartum have altered maternal lipid profile during early pregnancy [72].

$\mathrm{PE}$ is currently considered as a form of type 5 cardiorenal syndrome, an under-recognized entity in women's cardiovascular health $[73,74]$, which is associated with maternal endothelium alterations $[75,76]$. It seems that women at risk of PE have impaired utero-placental blood flow that may be associated with relatively hypoxic trophoblast that alters placental villous angiogenesis and produces abnormal vascular reactivity during gestation and metabolic changes [77-79]. From our results, it seems that some alterations persist for long period of time, even decades, after PE since hypertension, lipid metabolic alterations, altered body composition, hyperglycemia, and hyperinsulinemia were found in this systematic review.

Women with PE/E should be monitored and treated after delivery, including excessive body weight, metabolic alterations, hypertension and glucose and insulin disorders. It remains to be determined if postpartum and long term strict metabolic control intervention can reduce alterations found in this study in women with $\mathrm{PE} / \mathrm{E}$ in comparison to those without PE/E. Preventive clinical management should include screening and management of modifiable risk factors/outcomes and give healthy recommendation to neutralize negative changes demonstrated in this systematic review in women with PE/E. However, it remains to be determined if the alterations reported here are due to or are initiated by the PE/E phenomenon, or if the pregnancy findings are the result of a common cause in young women that is still present in older women.

\subsection{Strengths and limitations}

Our systematic review has several strengths: it was centered in PE/E, including women who suffered HELLP syndrome, without considering other hypertensive disorders of pregnancy which may have different 
A

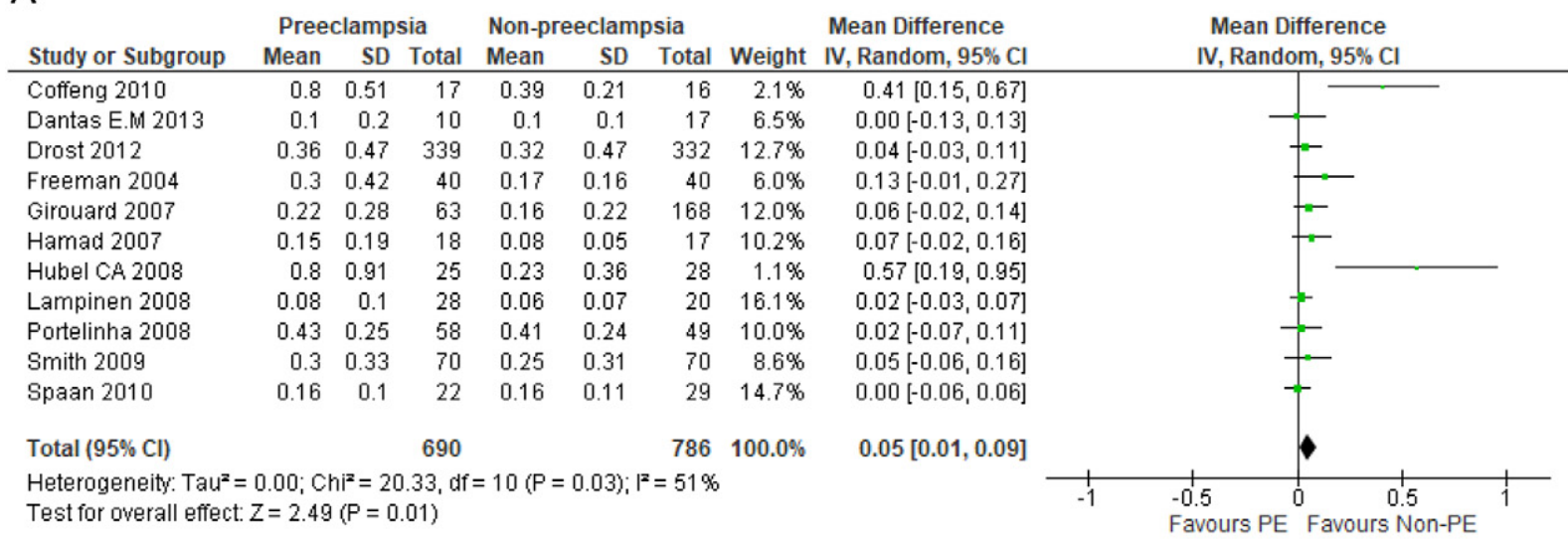

B

\begin{tabular}{|c|c|c|c|c|c|c|c|}
\hline Study or Subgroup & \multicolumn{2}{|c|}{ Preeclampsia } & \multicolumn{2}{|c|}{ Non-preeclampsia } & \multicolumn{2}{|r|}{ Risk Difference } & $\begin{array}{c}\text { Risk Difference } \\
\text { IV, Random, } 95 \% \mathrm{Cl}\end{array}$ \\
\hline Bar 1999 & 20 & 48 & 0 & 44 & $48.5 \%$ & $0.42[0.28,0.56]$ & \\
\hline McDonald 2013 & 9 & 109 & 10 & 219 & $51.5 \%$ & $0.04[-0.02,0.10]$ & \\
\hline Total $(95 \% \mathrm{Cl})$ & & 157 & & 263 & $100.0 \%$ & $0.22[-0.15,0.59]$ & \\
\hline Total events & 29 & & 10 & & & & \\
\hline $\begin{array}{l}\text { Heterogeneity: } \operatorname{Tau}^{2}= \\
\text { Test for overall effect }\end{array}$ & $\begin{array}{l}0.07 ; \mathrm{Chi}^{3} \\
\mathrm{Z}=1.16(\end{array}$ & $\begin{array}{l}23.63 \\
0.24)\end{array}$ & $d f=1(P<$ & 01); $1^{2}$ & $=96 \%$ & & 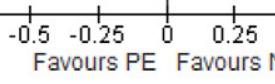 \\
\hline
\end{tabular}

C

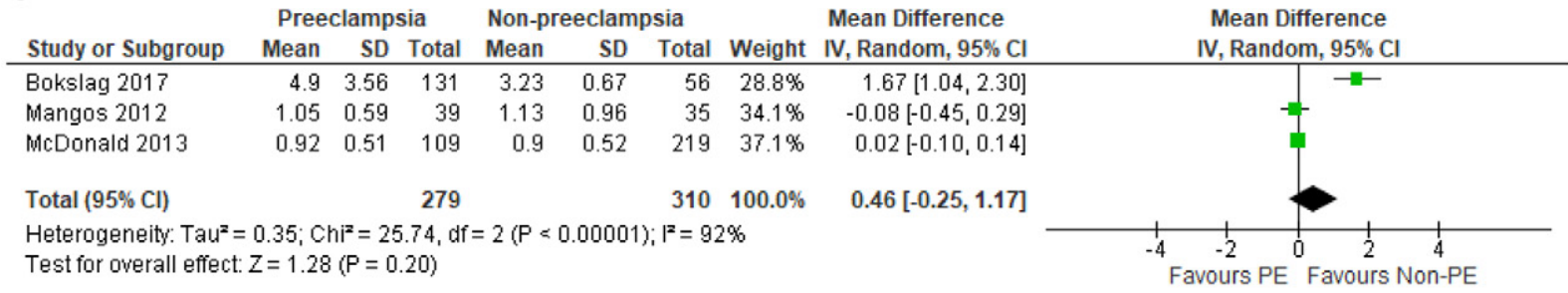

D

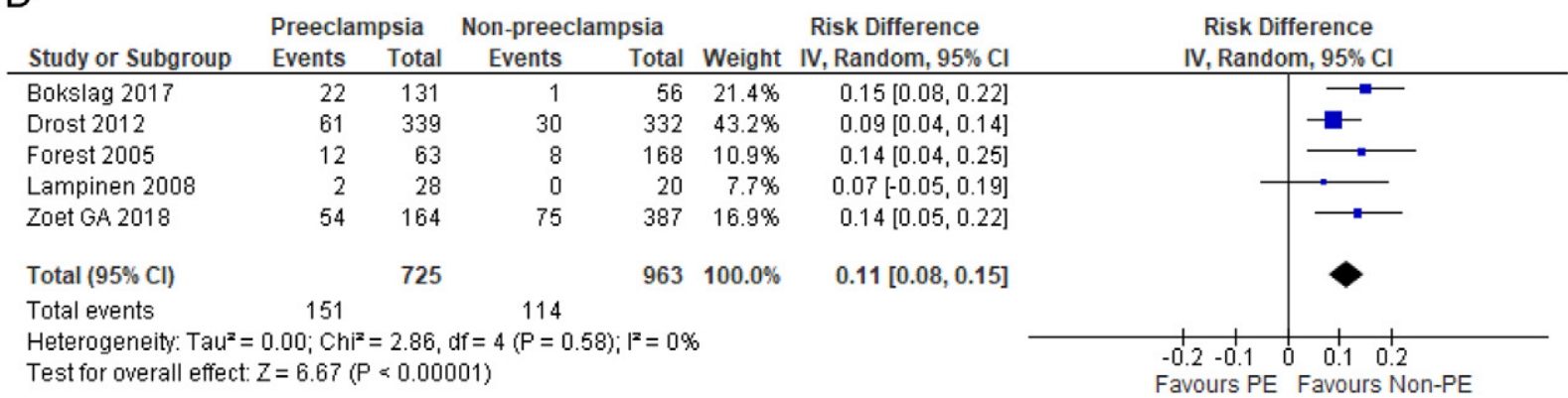

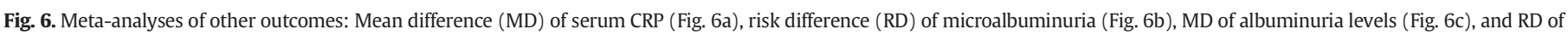

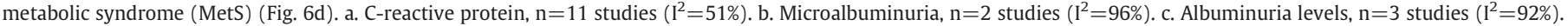
d. MetS, $\mathrm{n}=5$ studies $\left(\mathrm{I}^{2}=0 \%\right)$.

pathophysiology, organic causes and require different management to $\mathrm{PE} / \mathrm{E}$. We also evaluated several sources of heterogeneity of effects on outcomes across studies. Although strict diagnostic criteria were used in the conduct of the review, included studies were heterogeneous about intervals between pregnancy and later in life assessment and about information on lifestyle, nutrition and physical activity during the interval between pregnancy and later in life assessment. However, we could assume that these factors are similar in the normotensive pregnant women included as controls.

Subgroup analyses showed that effects were similar for studies with different intervals of time elapsed from pregnancies. Also, adjustment for confounding factors of the association between PE/E and outcomes, such as age and parity, was present in several studies; subgroup analyses by adjustment for confounders gave similar effects than studies without such adjustments. Finally, our study had other strengths including (i) exhaustive searches with low chances of selection bias; (ii) extractions were independent and double checked for accuracy with low risk of information bias; (iii) the majority of studies were of low risk of bias; and (iv) there was low publication bias.

Some limitations are worth to comment. Authors did not provide outcome data per PE and E separately, and PE/E treatment details were not described in most of studies. Also, MetS definitions were heterogeneous across studies, and may or not be based on published guidelines or consensus. 


\subsection{Interpretation}

PE and $\mathrm{E}$ are severe complications for both the mother and the fetus that sometimes should be controlled by termination of pregnancy $[1,5,6,71]$. The causes and triggers may be related abnormal maternal hepatic, vascular and kidney mechanisms or due to substances produced in the fetal compartment that secondarily alter different maternal organs and functions that are permanently affected in comparison to women without hypertension and proteinuria. Pregnant women should be monitored with anthropometric, metabolic and renal function assessment due to the increased cardiovascular, endocrine and metabolic risks.

This systematic review highlighted the close relationship between $\mathrm{PE} / \mathrm{E}$ and future metabolic, body composition and glucose/insulin markers, and MetS risks that might end up in future cardiovascular and endocrine disease, negative change in BMI and other intermediate markers. In the past few years, a link between PE/E with subclinical cardiorenal syndrome of pregnancy has been suggested as the main cause of that specific hypertensive syndrome of pregnancy [74].

\subsection{Conclusion}

$\mathrm{PE} / \mathrm{E}$ remains an under-recognized risk factor for future cardiovascular, metabolic, excessive BMI and kidney disease in women. In comparison to controls, $\mathrm{PE} / \mathrm{E}$ significantly increased systolic BP and diastolic BP, BMI, waist, waist-to-hip ratio, weight, total cholesterol, LDL, triglycerides, glucose, insulin, HOMA-IR index, C reactive protein, and the risks of hypertension and MetS. Also, PE/E reduced HDL levels. Heterogeneity of effects was high for most outcomes.

The close relationships between findings reported here with future health risk, the identification of markers of cardiovascular and metabolic risks may recommend a close clinical follow up of pregnant women with the alterations reported in women with PE/E. Rigorous interventions to prevent obesity, hypertension and other metabolic alterations in years after $\mathrm{PE} / \mathrm{E}$ pregnancy might provide clinical benefits although it remains to be determined decades after reproductive events.

\section{Funding}

This research did not receive any specific grant from funding agencies in the public, commercial, or not-for-profit sectors.

\section{Author contributions}

FRPL and AVH were involved in study conception and design; acquisition, and interpretation of data; drafting of the manuscript; and approval the final version of the manuscript.

VAV, YL, VP, and YMR were involved in acquisition and interpretation of data; and approval of the final version of the manuscript.

YL and AVH performed statistical analyses.

FRPL and AVH have access to the data and are responsible for the accuracy of the manuscript.

\section{Declaration of competing interest}

The authors declared no conflict of interest.

\section{Appendix A. Supplementary data}

Supplementary data to this article can be found online at https://doi. org/10.1016/j.metabol.2019.154012.

\section{References}

[1] August P, Sibai BM. Preeclampsia: clinical features and diagnosis. Uptodate 2019. https://www.uptodate.com/contents/preeclampsia-clinical-features-and-diagnosis.

[2] ACOG Practice Bulletin No. 202: gestational hypertension and preeclampsia. Obstet Gynecol 2019;133:e1-25. https://doi.org/10.1097/AOG.0000000000003018.

[3] Magee LA, Pels A, Helewa M, Rey E, von Dadelszen P. Canadian Hypertensive Disorders of Pregnancy Working Group. Diagnosis, evaluation, and management of the hypertensive disorders of pregnancy: executive summary. J Obstet Gynaecol Can 2014:36:416-41.

[4] Phoa KY, Chedraui P, Pérez-López FR, Wendte JF, Ghiabi S, Vrijkotte T, et al. Perinatal outcome in singleton pregnancies complicated with preeclampsia and eclampsia in Ecuador. J Obstet Gynaecol 2016;36:581-4.

[5] August P. Management of hypertension in pregnant and postpartum women Uptodate https://www.uptodate.com/contents/management-of-hypertension-inpregnant-and-postpartum-women; 2019.

[6] Antza C, Cifkova R, Kotsis V. Hypertensive complications of pregnancy: a clinical overview. Metab Clin Exp 2018;86:102-11.

[7] Jonsdottir LS, Arngrimsson R, Geirsson RT, Sigvaldason H, Sigfusson N. Death rates from ischemic heart disease in women with a history of hypertension in pregnancy. Acta Obstet Gynecol Scand 1995;74:772-6.

[8] Fraser A, Nelson SM, Macdonald-Wallis C. Associations of pregnancy complications with calculated cardiovascular disease risk and cardiovascular risk factors in middle age: the Avon Longitudinal Study of Parents and Children. Circulation 2012;125: 1367-80.

[9] Abhari FR, Ghanbari Andarieh M, Farokhfar A, Ahmady S. Estimating rate of insulin resistance in patients with preeclampsia using HOMA-IR index and comparison with non-preeclampsia pregnant women. Biomed Res Int 2014;2014: 1-9.

[10] Pauli JM, Preeclampsia RJT. Short-term and long-term implications. Obstet Gyneco Clin North Am 2015;42:299-313.

[11] Rana S, Lemoine E, Granger J, Karumanchi SA. Preeclampsia. Pathophysiology, challenges, and perspectives. Circ Res 2019;124:1094-112. https://doi.org/10.1161/ CIRCRESAHA.118.313276.

[12] Wu P, Haththotuwa R, Kwok CS, Babu A, Kotronias RA, Rushton C, et al. Preeclampsia and future cardiovascular health: a systematic review and meta-analysis. Circ Cardiovasc Qual Outcomes 2017;10. https://doi.org/10.1161/CIRCOUTCOMES.116. 003497 [pii: e003497].

[13] Wu P, Kwok CS, Haththotuwa R, Kotronias RA, Babu A, Fryer AA, et al. Pre-eclampsia is associated with a twofold increase in diabetes: a systematic review and metaanalysis. Diabetologia 2016;59:2518-26.

[14] Stroup DF, Berlin JA, Morton SC, et al. For the meta-analysis of observational studies in epidemiology (MOOSE) group. Meta-analysis of observational studies in epidemiology. A proposal for reporting. JAMA 2000;283:2008-12. https://doi.org/10.1001/ jama.283.15.2008.

[15] Tranquilli AL, Dekker G, Magee L, Roberts J, Sibai BM, Steyn W, et al. The classification, diagnosis and management of the hypertensive disorders of pregnancy: a revised statement from the ISSHP. Pregnancy Hypertens 2014;4:97-104. https://doi.org/10.1016/j.preghy.2014.02.001.

[16] Kattah AG, Garovic VD. The management of hypertension in pregnancy. Adv Chronic Kidney Dis 2013;20:229-39.

[17] ICD10Data.com Preeclampsia 014. https://www.icd10data.com/ICD10CM/ Codes/000-09A/010-016/014-International Classification of Diseases (ICD). https://www.who.int/classifications/icd/en/

[18] Peek MJ, Horvath JS, Child AG, Henderson-Smart DJ, Peat B, Gillin A. Maternal and neonatal outcome of patients classified according to the Australasian Society for the Study of Hypertension in Pregnancy Consensus Statement. Med J Aust 1995; 162:186-9.

[19] Newcastle-Otawa Scale. The Newcastle-Ottawa Scale (NOS) for assessing the quality of nonrandomised studies in meta-analyses. http://www.ohri.ca/programs/clinical_ epidemiology/oxford.asp (May 17, 2019).

[20] Hozo SP, Djulbegovic B, Hozo I. Estimating the mean and variance from the median, range, and the size of a sample. BMC Med Res Methodol 2005;5:13.

[21] Higgins JP. Commentary: heterogeneity in meta-analysis should be expected and appropriately quantified. Int J Epidemiol 2008;37:1158-60.

[22] Egger M, Davey Smith G, Schneider M, Minder C. Bias in metaanalysis detected by a simple, graphical test. BMJ 1997;315:629e34.

[23] Akhter T, Wikström AK, Larsson M, Naessen T. Individual artery wall layer dimensions indicate increased cardiovascular risk in previous severe preeclampsia -an investigation using non-invasive high-frequency ultrasound. Pregnancy Hypertens 2013;3(66). https://doi.org/10.1016/j.preghy.2013.04.027.

[24] Andersgaard AB, Acharya G, Mathiesen EB, Johnsen SH, Straume B, Øian P. Recurrence and long-term maternal health risks of hypertensive disorders of pregnancy: a population-based study. Am J Obstet Gynecol 2012;206:143.e1-8. https://doi. org/10.1016/j.ajog.2011.09.032.

[25] Aykas F, Solak Y, Erden A, Bulut K, Dogan S, Sarli B. Persistence of cardiovascular risk factors in women with previous preeclampsia: a long-term follow-up study. J Invest Med 2015;63:641-5. https://doi.org/10.1097/JIM.0000000000000189.

[26] Bar J, Kaplan B, Wittenberg C, Erman A, Boner G, Ben-Rafael Z, et al. Microalbuminuria after pregnancy complicated by pre-eclampsia. Nephrol Dial Transplant 1999;14:1129-32.

[27] Barden AE, Beilin LJ, Ritchie J, Walters BN, Michael C. Does a predisposition to the metabolic syndrome sensitize women to develop pre-eclampsia? J Hypertens 1999;17:1307-15.

[28] Berends AL, de Groot CJ, Sijbrands EJ, Sie MP, Benneheij SH, Pal R, et al. Shared constitutional risks for maternal vascular-related pregnancy complications and future 
cardiovascular disease. Hypertension 2008;51:1034-41. https://doi.org/10.1161/ HYPERTENSIONAHA.107.101873.

[29] Bokslag A, Teunissen PW, Franssen C, van Kesteren F, Kamp O, Ganzevoort W, et al. Effect of early-onset preeclampsia on cardiovascular risk in the fifth decade of life. Am J Obstet Gynecol 2017:216:523.e1-7. https://doi.org/10.1016/jajog 2017.02.015.

[30] Breetveld NM, Ghossein-Doha C, van Kuijk S, van Dijk AP, van der Vlugt MJ, Heidema WM, et al. Cardiovascular disease risk is only elevated in hypertensive, formerly preeclamptic women. BJOG 2015;122:1092-100. https://doi.org/10.1111/ 1471-0528.13057.

[31] Carleton H, Forsythe A, Flores R. Remote prognosis of preeclampsia in women 25 years old and younger. Am J Obstet Gynecol 1988;159:156-60

[32] Chambers JC. Fusi L, Malik IS, Haskard DO, De Swiet M, Kooner JS. Association of maternal endothelial dysfunction with preeclampsia. JAMA 2001;285:1607-12.

[33] Christensen M, Kronborg CS, Eldrup N, Rossen NB, Knudsen UB. Preeclampsia and cardiovascular disease risk assessment - do arterial stiffness and atherosclerosis uncover increased risk ten years after delivery? Pregnancy Hypertens 2016;6:110-4. https://doi.org/10.1016/j.preghy.2016.04.001.

[34] Coffeng SM, Blaauw J, Souwer ET, Rakhorst G, Smit AJ, Graaff R, et al. Skin autofluorescence as marker of tissue advanced glycation end-products accumulation in formerly preeclamptic women. Hypertens Pregnancy 2011;30:231-42. https://doi.org/10.3109/ 10641955.2010.484085.

[35] Dantas EM, Pereira FV, Queiroz JW, Dantas DL, Monteiro GR, Duggal P, et al. Preeclampsia is associated with increased maternal body weight in a northeastern Brazilian population. BMC Pregnancy Childbirth 2013;13:159. https://doi.org/10.1186 1471-2393-13-159.

[36] Drost JT, Arpaci G, Ottervanger JP, de Boer MJ, van Eyck J, van der Schouw YT, et al. Cardiovascular risk factors in women 10 years post early preeclampsia:the Preeclampsia Risk EValuation in FEMales study (PREVFEM). Eur J Prev Cardiol 2012; 19:1138-44. https://doi.org/10.1177/1741826711421079.

[37] Forest JC, Girouard J, Massé J, Moutquin JM, Kharfi A, Ness RB, et al. Early occurrence of metabolic syndrome after hypertension in pregnancy. Obstet Gynecol 2005;105 $1373-80$.

[38] Freeman DJ, McManus F, Brown EA, Cherry L, Norrie J, Ramsay JE, et al. Short- and long-term changes in plasma inflammatory markers associated with preeclampsia. Hypertension 2004;44:708-14.

[39] Garovic VD, Milic NM, Weissgerber TL, Mielke MM, Bailey KR, Lahr B, et al. Carotid artery intima-media thickness and subclinical atherosclerosis in women with remote histories of preeclampsia: results from a Rochester Epidemiology ProjectBased Study and Meta-analysis. Mayo Clin Proc 2017;92:1328-40. https://doi.org 10.1016/j.mayocp.2017.05.030

[40] Girouard J, Giguere Y, Moutquin JM, Forest JC. Previous hypertensive disease of pregnancy is associated with alterations of markers of insulin resistance. Hypertension 2007:49:1056-62.

[41] Hamad RR, Eriksson MJ, Silveira A, Hamsten A, Bremme K. Decreased flow-mediated dilation is present 1 year after a pre-eclamptic pregnancy. J Hypertens 2007;25 2301-7.

[42] He S, Silveira A, Hamsten A, Blombäck M, Bremme K. Haemostatic, endothelial and lipoprotein parameters and blood pressure levels in women with a history of preeclampsia. Thromb Haemost 1999;81:538-42.

43] Hubel CA, Powers RW, Snaedal S, Gammill HS, Ness RB, Roberts JM, et al. C-reactive protein is elevated 30 years after eclamptic pregnancy. Hypertension 2008;51: 1499-505. https://doi.org/10.1161/HYPERTENSIONAHA.108.109934.

[44] Innes KE, Weitzel L, Laudenslager M. Altered metabolic profiles among olde mothers with a history of preeclampsia. Gynecol Obstet Invest 2005:59:192-201.

[45] Kvehaugen AS, Andersen LF, Staff AC. Anthropometry and cardiovascular risk factors in women and offspring after pregnancies complicated by preeclampsia or diabetes mellitus. Acta Obstet Gynecol Scand 2010;89:1478-85. https://doi.org/10.3109/ 00016349.2010 .500368

[46] Laivuori H, Tikkanen MJ, Ylikorkala O. Hyperinsulinemia 17 years after preeclamptic first pregnancy. J Clin Endocrinol Metab 1996;81:2908-11.

[47] Lampinen KH, Rönnback M, Groop PH, Kaaja R]. A relationship between insulin sensitivity and vasodilation in women with a history of preeclamptic pregnancy. Hypertension 2008;52:394-401. https://doi.org/10.1161/HYPERTENSIONAHA.108.113423.

48] Mangos GJ, Spaan JJ, Pirabhahar S, Brown MA. Markers of cardiovascular disease risk after hypertension in pregnancy. J Hypertens 2012;30:351-8. https://doi.org/10. 1097/HJH.0b013e32834e5ac7.

[49] Manten GT, Sikkema MJ, Voorbij HA, Visser GH, Bruinse HW, Franx A. Risk factors for cardiovascular disease in women with a history of pregnancy complicated by preeclampsia or intrauterine growth restriction. Hypertens Pregnancy 2007;26:39-50

[50] McDonald SD, Ray J, Teo K, Jung H, Salehian O, Yusuf S, et al. Measures of cardiovascular risk and subclinical atherosclerosis in a cohort of women with a remote history of preeclampsia. Atherosclerosis 2013;229:234-9. https://doi.org/10.1016/j.atherosclerosis.2013.04.020.

[51] Nisell H, Erikssen C, Persson B, Carlström K. Is carbohydrate metabolism altered among women who have undergone a preeclamptic pregnancy? Gynecol Obstet Invest 1999;48:241-6.

[52] Nohira T. Hypertension and metabolic abnormalities later in life after preeclampsia. Hypertens Res Pregnancy 2013;1:52-6.

[53] Östlund E, Al-Nashi M, Hamad RR, Larsson A, Eriksson M, Bremme K, et al. Normalized endothelial function but sustained cardiovascular risk profile 11 years following a pregnancy complicated by preeclampsia. Hypertens Res 2013;36:1081-7. https://doi.org/ 10.1038/hr.2013.81

[54] Portelinha A, Belo L, Tejera E, Rebelo I. Adhesion molecules (VCAM-1 and ICAM-1) and C-reactive protein in women with history of preeclampsia. Acta Obstet Gynecol Scand 2008;87:969-71. https://doi.org/10.1080/00016340802322265.
[55] Portelinha A, Belo L, Cerdeira AS, Braga J. Tejera E, Pinto F, et al. Lipid levels including oxidized LDL in women with history of preeclampsia. Hypertens Pregnancy 2010; 29:93-100. https://doi.org/10.3109/10641950902968593.

[56] Pouta A, Hartikainen AL, Sovio U, Gissler M, Laitinen J, McCarthy MI, et al. Manifestations of metabolic syndrome after hypertensive pregnancy. Hypertension 2004; 43:825-31.

[57] Romundstad PR, Magnussen EB, Smith GD, Vatten LJ. Hypertension in pregnancy and later cardiovascular risk: common antecedents? Circulation 2010;122: 579-84. https://doi.org/10.1161/CIRCULATIONAHA.110.943407.

[58] Sattar N, Ramsay J, Crawford L, Cheyne H, Greer IA. Classic and novel risk factor parameters in women with a history of preeclampsia. Hypertension 2003;42: 39-42.

[59] Smith GN, Walker MC, Liu A, Wen SW, Swansburg M, Ramshaw H, et al. Pre-Eclampsia New Emerging Team (PE-NET). A history of preeclampsia identifies women who have underlying cardiovascular risk factors. Am J Obstet Gynecol 2009;200(58): e1-8. https://doi.org/10.1016/j.ajog.2008.06.035.

[60] Spaan JJ, Houben AJ, Musella A, Ekhart T, Spaanderman ME, et al. Insulin resistance relates to microvascular reactivity 23 years after preeclampsia. Microvasc Res 2010;80:417-21.

[61] Suzuki H, Watanabe Y, Arima H, Kobayashi K, Ohno Y, Kanno Y. Short- and long-term prognosis of blood pressure and kidney disease in women with a past history of preeclampsia. Clin Exp Nephrol 2008;12:102-9. https://doi.org/10.1007/s10157-0070018-1.

[62] White WM, Mielke MM, Araoz PA, Lahr BD, Bailey KR, Jayachandran M, et al. A history of preeclampsia is associated with a risk for coronary artery calcification 3 decades later. Am J Obstet Gynecol. 2016; 214:519.e1-519.e8. https://doi.org/10. 1016/j.ajog.2016.02.003.

[63] Zoet GA, Benschop L, Boersma E, Budde RPJ, Fauser BCJM, van der Graaf Y, et al. CREW Consortium. Prevalence of subclinical coronary artery disease assessed by coronary computed tomography angiography in 45- to 55-year-old women with a history of preeclampsia. Circulation 2018;137:877-9. https://doi.org/10.1161/ CIRCULATIONAHA.117.032695.

[64] Hernández-Díaz S, Toh S, Cnattingius S. Risk of pre-eclampsia in first and subsequent pregnancies: prospective cohort study. BMJ 2009;338:b2255. https://doi.org/10. 1136/bmj.b2255.

[65] Bateman BT, Bansil P. Hernandez-Diaz S, Mhyre JM, Callaghan WM, Kuklina EV. Prevalence, trends, and outcomes of chronic hypertension: a nationwide sample of delivery admissions. Am J Obstet Gynecol 2012 Feb;206(2):134.e1-8. https://doi.org/10.1016/j. ajog.2011.10.878

[66] MacDonald EJ, Lepine S, Pledger M, Geller SE, Lawton B, Stone P. Pre-eclampsia causing severe maternal morbidity - a national retrospective review of preventability and opportunities for improved care. Aust N Z J Obstet Gynaecol 2019 Mar 18. https://doi.org/10.1111/ajo.12971.

[67] Harville EW, Viikari JS, Raitakari OT. Preconception cardiovascular risk factors and pregnancy outcome. Epidemiology 2011;22:724-30. https://doi.org/10.1097/EDE. 0b013e318225c960.

[68] Magnussen EB, Vatten LJ, Smith GD, Romundstad PR. Hypertensive disorders in pregnancy and subsequently measured cardiovascular risk factors. Obstet Gynecol 2009;114:961-70. https://doi.org/10.1097/AOG.0b013e3181bb0dfc.

[69] Brown MC, Best KE, Pearce MS, Waugh J, Robson SC, Bell R. Cardiovascular disease risk in women with pre-eclampsia: systematic review and meta-analysis. Eur J Epidemiol 2013;28:1-19. https://doi.org/10.1007/s10654-013-9762-6.

[70] Covella B, Vinturache AE, Cabiddu G, Attini R, Gesualdo L, Versino E, et al. A systematic review and meta-analysis indicates long-term risk of chronic and end-stage kidney disease after preeclampsia. Kidney Int 2019;96:711-27. https://doi.org/10.1016/j.kint. 2019.03.033.

[71] Alese MO, Moodley J Naicker T. Preeclampsia and HELLP syndrome, the role of the liver. J Matern Fetal Neonatal Med 2019 Jan;31:1-7. https://doi.org/10.1080/ 14767058.2019.1572737.

[72] Adank MC, Benschop L, Peterbroers KR, Smak Gregoor AM, Kors AW, Mulder MT, et al. Is maternal lipid profile in early pregnancy associated with pregnancy complications and blood pressure in pregnancy and long term postpartum? Am J Obstet Gynecol 2019;221:150.e1-150.e13. https://doi.org/10.1016/j.ajog.2019. 03.025

[73] Rangaswami J, Naranjo M, McCullough PA. Preeclampsia as a form of type 5 cardiorenal syndrome: an underrecognized entity in women's cardiovascular health. Cardiorenal Med 2018;8:160-72. https://doi.org/10.1159/ 000487646.

[74] Gyselaers W, Thilaganathan B. Preeclampsia: a gestational cardiorenal syndrome. J Physiol 2019. https://doi.org/10.1113/jp274893.

[75] Powe CE, Levine RJ, Karumanchi SA. Preeclampsia, a disease of the maternal endothelium: the role of antiangiogenic factors and implications for later cardiovascular disease. Circulation 2011;123:2856-69.

[76] Benschop L, Schalekamp-Timmermans S, Schelling SJC, Steegers EAP, Roeters van Lennep JE. Early pregnancy cardiovascular health and subclinical atherosclerosis. J Am Heart Assoc 2019 Aug 6;8(15):e011394. https://doi.org/10.1161/JAHA.118.011394.

[77] Hübinette A, Lichtenstein P, Brismar K, Vatten L, Jacobsen G, Ekbom A, Cnattingius S. Serum insulin-like growth factors in normal pregnancy and in pregnancies complicated by preeclampsia. Acta Obstet Gynecol Scand 2003 Nov;82(11):1004-9. PubMed PMID: [14616273].

[78] Ingec M, Gursoy HG, Yildiz L, Kumtepe Y, Kadanali S. Serum levels of insulin, IGF-1, and IGFBP-1 in pre-eclampsia and eclampsia. Int J Gynaecol Obstet 2004:84(3):2149. PubMed PMID: 15001368.

[79] Burton GJ, Redman CW, Roberts JM, Moffett A. Pre-eclampsia: pathophysiology and clinical implications. BM] 2019;366. https://doi.org/10.1136/bmj.I2381. 